\title{
The surface brightness of the Galaxy at the solar neighbourhood ${ }^{\star}$
}

\author{
A.-L. Melchior ${ }^{1}$, F. Combes ${ }^{1}$, and A. Gould ${ }^{2, \star \star}$ \\ 1 LERMA, Université Pierre et Marie Curie and Observatoire de Paris, 61 avenue de l'Observatoire, 75014 Paris, France \\ e-mail: Anne-Laure.Melchior@obspm.fr; Francoise.Combes@obspm.fr \\ 2 Department of Astronomy, Ohio State University, Columbus Ohio 43210, USA \\ e-mail: gould@astronomy.ohio-state.edu
}

Received 4 August 2006 / Accepted 2 November 2006

\section{ABSTRACT}

\begin{abstract}
We present a new determination of the surface brightness of our Galaxy at the Solar Neighbourhood as observed from outside the Galaxy. We rely on various existing optical and infra-red surveys to obtain a multiwavelength estimate. On the one hand, scattered light does not contribute significantly to the surface brightness. On the other hand, optical and infrared integrated all-sky surveys (Pioneer 10/11 and COBE/DIRBE) show a systematically larger value than our synthetic local estimate based on Hipparcos data. This local estimate is also compatible with our Galactic simulations normalised at the Solar Neighbourhood and assuming a homogeneous stellar distribution. We interpret this disagreement as a signature of the presence of a local minimum of the stellar density compatible with Gould's belt. According to this result, the global luminosity of the Milky Way should follow the Tully-Fisher relation established for external galaxies.
\end{abstract}

Key words. Galaxy: solar neighbourhood - cosmology: diffuse radiation - radiation mechanisms: thermal - methods: data analysis ISM: general

\section{Introduction}

Modelling of the multiwavelength continuum emission of high$z$ galaxies is essential to simulate realistically the formation and evolution of galaxies as will be observed with the next generation of instruments. The data samples of galaxies are currently limited especially in the infrared and millimeter wavelengths, but will be significantly enlarged in the coming years. On the one hand, theoretical modelling based on physical principles actually relies on a large number of variables and requires wellsampled galaxy spectra to be constrained. On the other hand, empirical approaches (e.g. Guiderdoni et al. 1998; Dale et al. 2001) minimise the number of parameters to reproduce the current observations. Both approaches need to be validated with well-sampled continuum spectra of galaxies. It is customary to use the "Solar Neighbourhood" as a zero-point for these models. Even though various multiwavelength observations are available for the Galaxy and the Solar Neighbourhood, most authors quote Mathis et al. (1981), who were investigating the Local Interstellar Radiation Field. Although this work is sufficiently robust to understand the absorption of photons by dust grains in the Local Neighbourhood, it did not intend to provide a spectral measurement of the surface brightness of our Galaxy at the Solar Neighbourhood as observed from outside the Galaxy.

In this paper, we compile various data sets to measure this quantity, which could be used as a zero-point for spectral modelling of external galaxies. We perform a cosecant averaging based on a plane parallel approximation for this measurement.

\footnotetext{
* Appendices are only available in electronic form at http: //www . aanda.org

$\star \star$ Visiting Astronomer, LPCC, Collège de France, 11 place Marcelin Berthelot, 75231 Paris, France.
}

In Sect. 2, we explain the basis for this approximation and its expected behaviour. We compare this approximation (integration in a cone) with the method usually used for external galaxies (integration in a cylinder) through simulations. In Sect. 3, we present a synthetic approach based on the Hipparcos data set and a stellar spectral flux library to estimate an optical spectrum of surface brightness of our Galaxy. We also present the two different types of integration (cone/cylinder), described here as corrected direct and indirect methods. In Sects. 4 and 5, we present a compilation of various all-sky surveys with the cosecant averaging method. In the optical, we use the stellar catalogues Tycho-2 and USNO-A2 as well as the Pioneer 10/11 integrated maps. In the infra-red, we project in cosec $b$ the COBE/DIRBE survey. Last, we discuss all these estimates.

\section{Methods and simulations}

The purpose of this paper is to determine at different wavelengths the surface brightness of our Galaxy at the Solar Neighbourhood as observed from outside the Galaxy (cf. Appendix B for a formal definition). However, such an observational measurement imposes strong constraints: (1) we want to compare different wavelengths (which could be sampled differently); (2) we would prefer to avoid the Galactic disc area, which is affected by crowding (stellar catalogues); (3) we would like to sample a significant volume in order to reduce local fluctuations.

The available all-sky surveys consist of stellar catalogues or integrated maps, at different wavelengths. In order to minimise the systematics due to this diversity, we adopt the same averaging procedure for each data set. The idea is to apply a cosecant averaging on the data to estimate the surface brightness of the 
Galaxy. This method, also called integration in a "cone", satisfies the previous constraints, while approximating the integration in a cylinder, usually used to compute surface brightness of external galaxies. In this section, we present the different aspects of this approximation with respect to the standard method. Firstly, we present the modelling performed for our Galaxy and the methods used to compute the surface brightness. We describe the "integration in a cylinder", which corresponds to the standard method to compute a surface brightness. We explain the difficulties due to our position near the plane of the Galaxy, and the corrections required to estimate the surface brightness corresponding to a face-on observation. In parallel, we present the alternate method used throughout this paper, called here "integration in a cone". We discuss and compare the trends of both. Secondly, we simulate the $\operatorname{cosec} b$ projection that will be applied to stellar catalogues.

\subsection{Definitions}

We consider two extreme geometries for our Galaxy: (1) an exponential disc with scale length $h_{R}=2.5 \mathrm{kpc}$ (Robin et al. 1992) and (variable) scale height ${ }^{1} h_{Z}\left(M_{V}\right)$, with a density normalised at the Solar neighbourhood; (2) a plane parallel model with a constant number density and a total thickness of $2 h_{Z}=240 \mathrm{pc}$. In both cases, we assume that the Sun is located at a cylindrical Galactocentric radius $r_{q}=R_{\odot}=8 \mathrm{kpc}$, at $z_{\odot}=15 \mathrm{pc}$ above the Galactic plane (Humphreys \& Larsen 1995) and that the total extension of the Galaxy with respect to the centre is $R_{\mathrm{gal}}=14 \mathrm{kpc}$. The numbers $n\left(r, b, l, M_{V}\right)$ of $M_{V}$ stars per $\mathrm{pc}^{3}$ at the position $(r, b, l)$ for the 2 geometries are as follows:

$$
\begin{aligned}
n^{\text {D.Expon. }} & \left(r, b, l, M_{V}\right)=n_{\odot}\left(M_{V}\right) \exp \left(z_{\odot} / h_{Z}\left(M_{V}\right)\right) \\
& \exp \left(R_{\odot} / h_{R}\right) \exp \left(-r_{q} / h_{R}\right) \exp \left(-\left|z+z_{\odot}\right| / h_{Z}\left(M_{V}\right)\right)
\end{aligned}
$$

with the cylindrical Galactocentric radius $r_{q}=\left(r_{\odot}^{2}+r^{2} \cos b^{2}-\right.$ $\left.2 r_{\odot} r \cos b \cos l\right)^{1 / 2}$; the number density of $M_{V}$ stars per $\mathrm{pc}^{3}$ $n_{\odot}\left(M_{V}\right)=\phi\left(M_{V}\right)$ is the luminosity function $\phi\left(M_{V}\right)$ at the Solar Neighbourhood, as defined in Appendix D; $z=r \sin b$ is the vertical position with respect to the Sun;

$n^{P P}\left(r, b, l, M_{V}\right)= \begin{cases}n_{\odot}\left(M_{V}\right) \exp \left(z_{\odot} / h_{Z}\left(M_{V}\right)\right) & \text { if }\left|z+z_{\odot}\right|<h_{Z}, \\ 0 & \text { if }\left|z+z_{\odot}\right| \geq h_{Z} .\end{cases}$

We account for extinction with a uniform dust layer following the prescription provided and discussed in Appendix E.

\subsubsection{Integration in a cylinder}

We define the average surface brightness $v S_{v}$ of our Galaxy at the Solar neighbourhood in the $V$ band.

$$
\begin{aligned}
v_{V} S_{V}= & {\left[\int_{r, b, l, M_{V}} n\left(r, b, l, M_{V}\right) f\left(A_{V}(r, b)\right) L\left(M_{V}\right)\right.} \\
& \left.r^{2} \cos b \mathrm{~d} r \mathrm{~d} b \mathrm{~d} l \mathrm{~d} M_{V}\right]_{r_{q}<R_{\mathrm{cyl}}} / \pi R_{\mathrm{cyl}}^{2}
\end{aligned}
$$

where $L\left(M_{V}\right)$ is the luminosity ${ }^{2}$ of a star of $M_{V}$ magnitude, $f\left(A_{V}(r, b)\right)=10^{-0.4 A_{V}(r, b)}$ is the extinction factor and $R_{\text {cyl }}$ is the

\footnotetext{
${ }^{1}$ Similarly to Miller \& Scalo (1979), we take $h_{Z}=80 \mathrm{pc}$ for $M_{V} \leq 0$, $h_{Z}=250 \mathrm{pc}$ for $M_{V} \geq 8$, and a linear interpolation in between. We also consider a fixed scale height $\left(h_{Z}=120 \mathrm{pc}\right)$, compatible with the variable scale height modelling in Fig. 4. See also Appendix D.

${ }^{2}$ We derive these luminosities in $V$ with the formula $L\left(M_{V}\right)=$ $C_{V} 10^{-0.4 M_{V}} \times 4 \pi\left(D_{(=10 \mathrm{pc})}\right)^{2}$, where $C_{V}=1.97 \times 10^{-8} \mathrm{~W} \mathrm{~m}^{-2}$.
}

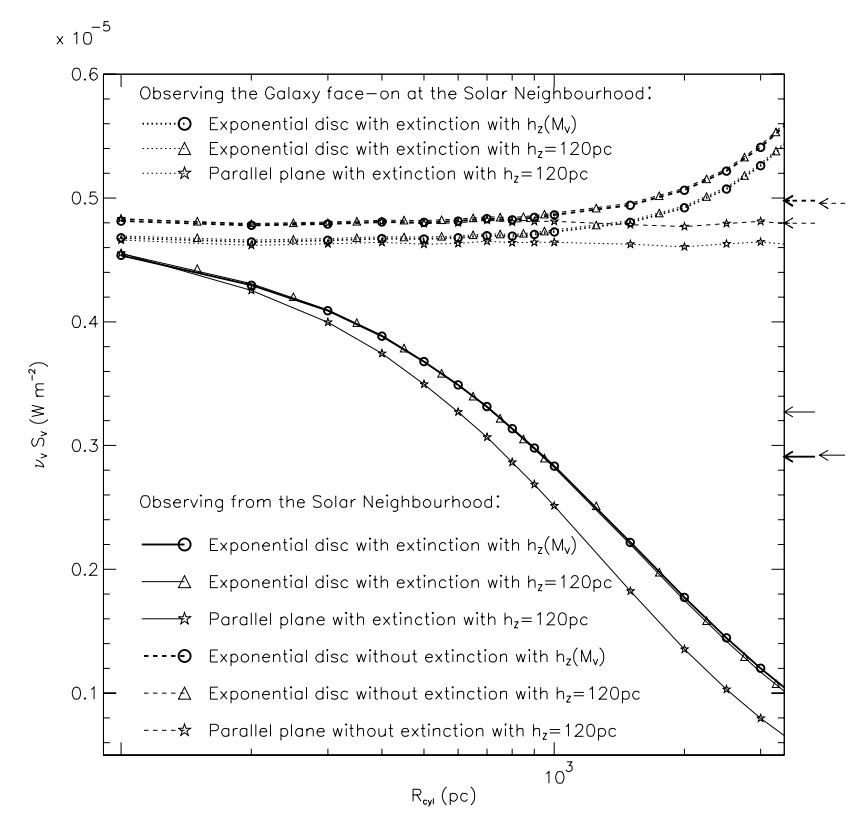

Fig. 1. Simulations. Surface Brightness in $V$ band of the Milky Way as a function of the cylinder radius $R_{\text {cyl }}$ for the models of Sect. 2 . The cylinders corresponding to $R_{\text {cyl }}$ are centered on the Sun and perpendicular to the Galactic plane. The curves with symbols correspond to the surface brightness computed with a cylinder for the exponential disc or plane parallel geometry. We consider two different observers: one observing from the Solar Neighbourhood and one observing the Galaxy face-on. We compare an ideal galaxy without extinction with the real configuration with extinction. The arrows on the right-hand side correspond to the integration in a cone with the cosecant law approximation, fitted for $1 / \sin |b| \leq 6$. The caption for the line sizes (and types) of these horizontal lines are defined in Fig. 4.

radius of the cylinder used to compute the surface brightness (see Fig. 1). In the absence of extinction and for $R_{\text {cyl }}<2 \mathrm{kpc}$, the two geometries are equivalent and the surface brightness is nearly independent of the cylinder radius. With extinction, the surface brightness measured from the Solar Neighbourhood decreases with increasing cylinder radius. This is due to the extinction affecting the $|b|<90$ deg lines of sight crossing the dust plane. Of course, this effect is not observed when one computes the surface brightness for an observer of the face-on Galaxy, as only the lines of $\operatorname{sight}^{3}(|b|=90 \mathrm{deg})$ perpendicular to the Galactic plane are integrated. In Fig. 2, we display the correction factor necessary to convert our local estimates (measured from the Solar Neighbourhood) into surface brightness of the face-on Galaxy (see also Table E.1).

\subsubsection{Integration in a "cone" - cosecant-law approximation}

We integrate the flux received at the Sun in a cone, according to the cosecant-law approximation (see Fig. 3). The slope of the corresponding function $v_{\lambda} I_{\lambda}(|b|)$ is the surface brightness of our Galaxy defined above, assuming a homogeneous stellar density galaxy.

$$
\begin{aligned}
v_{V} I_{V}(|b|)= & 2 \times \int_{r, l, M_{V}} n\left(r, b, l, M_{V}\right) \\
& \frac{f\left(A_{V}(r, b)\right) L\left(M_{V}\right)}{4 \pi} \cos b \mathrm{~d} b \mathrm{~d} r \mathrm{~d} l \mathrm{~d} M_{V}
\end{aligned}
$$

${ }^{3}$ For our Galaxy, the extinction corresponding to the total disc thickness is $0.12 \mathrm{Vmag}$. 


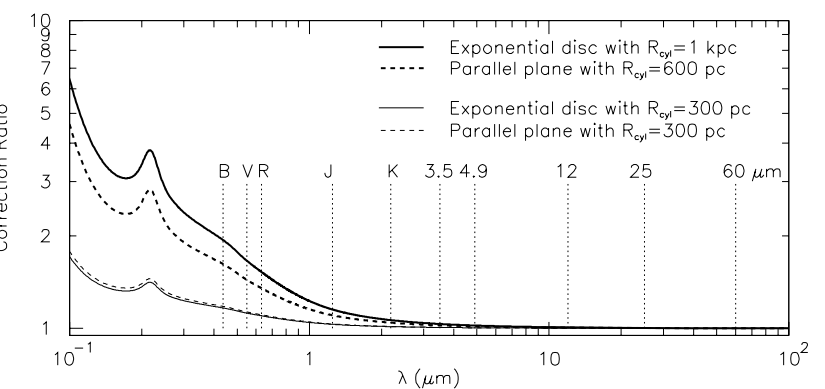

Fig. 2. Correction ratio as a function of wavelength necessary to derive the surface brightness of the Galaxy (observed face-on) from observations at the Solar Neighbourhood. We display the corrections (thick lines) for a cylinder of radius $1 \mathrm{kpc}$ and $600 \mathrm{pc}$ (corresponding to the $1 / \sin b<6$ cut for the cosecant law approximation). We also provide for comparison purposes the corresponding curves (thin lines) for a cylinder radius of $300 \mathrm{pc}$. For the wavelength dependence of the extinction, we assume $R_{V}=3.1$ and use the prescription of Fitzpatrick (1999). The different wavelengths for which we project in $\operatorname{cosec} b$ all-sky data are indicated (and numerical values provided in Table E.1).

The normalisation factor in front of Eq. (4) is due to the normalisation of this method with respect to the integration in a cylinder. As explained in Appendix $\mathrm{C}$, it has been determined for a uniform plane parallel geometry. Figure 4 displays the $v_{V} I_{V}(|b|)$ function, used to compute the surface brightness. To a good approximation, the curves are linear.

In the case of a plane parallel approximation without extinction, the slope of the $I_{V}(b)$ function with respect to $1 / \sin b$ is equal to the value at $b=90 \mathrm{deg}$, namely:

$\frac{I_{V}(b)-I_{V}(b=90 \mathrm{deg})}{1 / \sin b-1} \sim I_{V}(b=90 \mathrm{deg})$.

For a linear function, Eq. (5) is an equality and is equivalent to $v_{V} S_{V}=I_{V}(b=90 \mathrm{deg})=I_{V}(b) \sin b$, where $v_{V} I_{V}(b=90 \mathrm{deg})$ corresponds to the surface brightness. Figure 4 shows that, without extinction, the exponential disc geometry is very close to this approximation. In the presence of extinction, the two geometries (plane parallel and exponential disc) are also very close. Figure 5 displays the sensitivity of the cosecant-law approximation to the cut in $(1 / \sin |b|)_{\text {MAX }}$.

\subsubsection{Comparison}

Plane parallel and exponential disc geometries provide very similar results. In Fig. 1, we compare the two modes of integration in $V$.

In the absence of extinction, the two modes of integration (in a cone and in a cylinder) are equivalent for $R_{\text {cyl }}<2 \mathrm{kpc}$. With extinction, a flattening is observed for both methods. For integration in a cone, it is sensitive to the cut in $1 / \sin b$ used to compute the slope (see Fig. 5). For integration in a cylinder, the surface brightness decreases as the radius of the cylinder considered increases (see Fig. 1).

As displayed in Fig. 1, we estimate that $1 / \sin |b| \leq 6$ is the best compromise to sample the surface brightness of the Solar Neighbourhood over the whole spectral range. The cosecantlaw approximation (computed with $1 / \sin |b| \leq 6$ ) is compatible with the surface brightness computed in a cylinder of radius $1 \mathrm{kpc}$. The plane parallel model provides a similar equivalence at $600 \mathrm{pc}$. For this simple geometry, this radius can be compared to the maximum radius probed by the integration in a cone: $h_{Z} / \tan b_{\max }$. For the parameter values considered, stars up to a radius of $710 \mathrm{pc}$ are probed. For the exponential disc, we expect a similar difference (but asymmetric).

Table 1 provides the values thus obtained for the $V$ surface brightness of our Galaxy at the Solar Neighbourhood for the different cases. It is important to note that the difference between the surface brightness estimated without extinction and the faceon Galaxy surface brightness (with extinction) is so small that scattered light can be neglected (less than $6 \%$ of the surface brightness).

\subsection{Simulation of a catalogue reduction (observational procedure)}

In the following, we will compile several whole sky stellar catalogues to measure the surface brightness of the Solar Neighbourhood. In order to understand the possible trends that could affect this observational procedure, we simulate this reduction for a $V$ catalogue based on the cosecant-law approximation. We take our exponential geometry model with extinction and a variable scale height $h_{Z}\left(M_{V}\right)$ and decompose the $I(|b|)$ function into apparent $V$ magnitude bins. We also divide the sky into 4 areas, in order to study asymmetries between the North and South Galactic hemispheres and the Galactic Centre and AntiCentre directions. The sum of the slope of each bin provides the surface brightness at the Solar Neighbourhood.

Figures 6 and 7 display this simulation for $V<6$ and $V \geq 7$ stars. The flux is higher in the direction of Galactic Centre. We observe a significant flattening for large $1 / \sin |b|$, due to extinction. We also clearly detect the asymmetric position of the Sun above the Galactic plane $\left(z_{\odot}=15 \mathrm{pc}\right)$. The left (resp. right) panel of Fig. 8 summarises the contribution of each apparent $V$ magnitude bin to the surface brightness for $h_{Z}\left(M_{V}\right)$ (resp. $h_{Z}=120 \mathrm{pc}$ ). Table A.1 summarises the corresponding contribution per apparent magnitude bins. It also provides the fitted value at $b=90^{\circ}$.

Each bin with $V<2$ and $V>21$ stars contributes to no more than about $1 \%$ of the surface brightness, while the main contributors ( $86.5 \%$ of the total surface brightness) are $5<V<$ 16 stars. Uncertainties on the very bright stars, which are often missed in stellar catalogues (due to their saturation, extinction in the stellar disc, etc.), and dim stars (close to the detection limit) will not bias the surface brightness estimate.

Note that this method, uncorrected for extinction, provides an estimate of the surface brightness computed from the Solar Neighbourhood, and has to be corrected as indicated in Fig. 2 and Table E. 1 to obtain the face-on value.

\section{Stellar surface brightness: a synthetic approach with the Hipparcos data}

The Hipparcos Catalogue (Perryman et al. 1997; van Leeuwen et al. 1997) with the Hipparcos Input Catalogue (HIC) (Turon et al. 1992, 1995) constitute a unique database to explore the Solar Neighbourhood. Spectral type, parallax, and $V$ magnitude are provided for each $V<7.3$ star. We restrict the sample to 13374 stars with a $3 \sigma$ parallax and with the constraints on the completion limits described in Appendix D. In the following, we use the stellar spectral flux library from Pickles (1998) to produce synthetic spectra: a spectrum is associated with each star and normalised to the observed $V$ flux. Hipparcos stars are then combined in two different ways to get estimates of the surface brightness. In Sect. 3.1, we directly sum the flux of the observed stars with a $\sin |b|$ weighting. However, this direct 


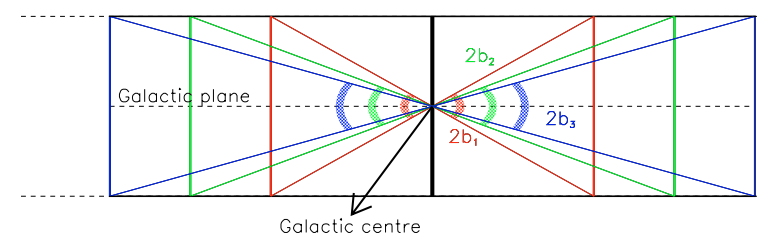

(a)

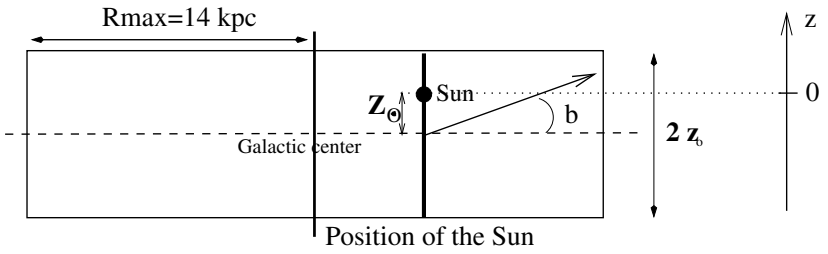

(b)

Fig. 3. Plane parallel approximation and cosecant method. The matter (HI gas or stars for instance) is assumed to be organised in planes parallel to the Galactic plane. a) We assume an infinite thick sheet of uniform density centred on the Galactic plane. In this configuration, the column density perpendicular to the plane can be derived from the integration of the column density along various directions $b=b_{1}, b_{2}$ or $b_{3} \ldots$ as defined in Eq. (5). b) In this paper, we consider a truncated sheet for the plane parallel configuration, and then study this approximation for an exponential disc.

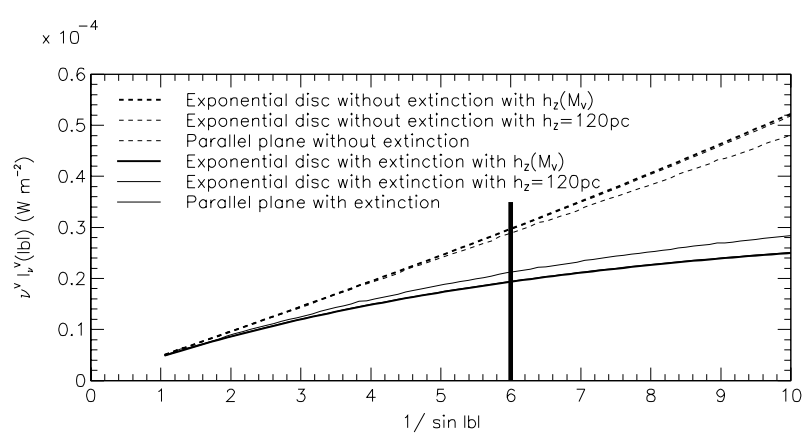

Fig. 4. Simulations. We present the cosecant laws computed for exponential disc and plane parallel geometries. We also consider the effect of extinction. When not indicated otherwise, the slopes are measured for $1 / \sin |b|<6$. The fixed and variable $h_{z}$ geometries are barely distinguishable.

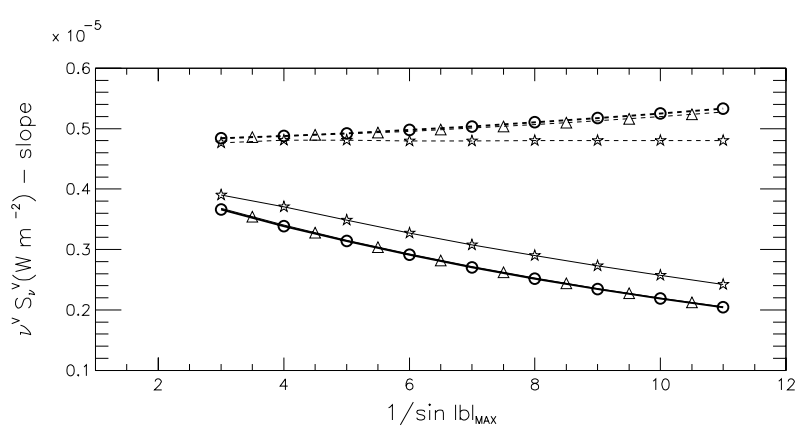

Fig. 5. Simulations. $V$ Surface Brightness derived from the cosecant laws as a function of the chosen cut in $1 / \sin |b|$. The symbols and lines follow the same convention as Fig. 1. The line widths and types are also defined in Fig. 4.

measurement requires a volume correction due to the apparent magnitude limit of the sample used. This corrected direct measurement corresponds to the cosecant averaging (integration in a cone). In Sect. 3.2, we compute the surface brightness with the luminosity of the stars with the integration in the cylinder approach. Appropriate volume corrections are also required.

\subsection{Corrected direct measurement of starlight: a synthetic spectrum}

The cosecant-law approximation (see Eq. (5)) enables to compute directly the surface brightness as the sum of the stellar fluxes weighted by $\sin |b|$. Even though the amount of dust is small at the Solar neighbourhood, it significantly affects the surface brightness computation (see Fig. 1). We have shown in
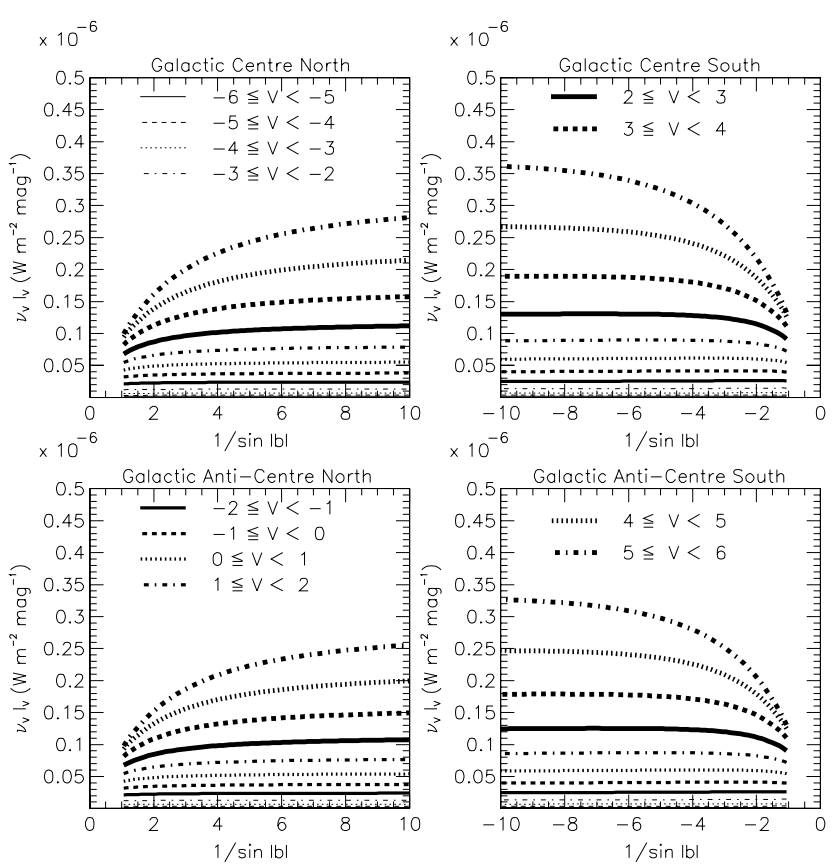

Fig. 6. Simulations in $V$ with extinction. Integrated $V$ flux in 1 mag bins as a function of $1 / \sin |b|$ for the bright $\operatorname{stars}(V<6)$.

Table 1. Simulated $V$ surface brightness expressed in different units. The first and second columns provide the surface brightness estimates derived from the Solar Neighbourhood (S.N.) and for the face-on Galaxy observed at the Solar Neighbourhood. The third column provides the estimate in the absence of extinction. The last column gives the unit of value provided in the different lines.

\begin{tabular}{rrrl}
\hline \hline from S.N. & Face-on @ S.N. & without extinction & units \\
\hline $2.91 \times 10^{-6}$ & $4.72 \times 10^{-6}$ & $4.98 \times 10^{-6}$ & $\mathrm{~W} \mathrm{~m}^{-2}$ \\
9.7 & 15.6 & 16.5 & $L_{\odot} \mathrm{pc}^{-2}$ \\
23.90 & 23.37 & 23.31 & $\mathrm{mag} \mathrm{arcsec}^{-2}$ \\
\hline
\end{tabular}

Sect. 2 that the surface brightness of our Galaxy observed faceon is very close to the surface brightness computed for a Galaxy without dust. Hence, we subtract the extinction to each star relying on our dust modelling (see Appendix E), and obtain the lower bound:

$$
\begin{aligned}
v S_{v} & >2 \times \sum_{i}^{\text {all stars }} v F_{v}\left(M_{V}, S p T\right) / f\left(A_{V}\right) \times \sin |b| \\
& >2 \times \sum_{i}^{\text {all stars }} \frac{L_{\lambda}\left(M_{V}, S p T\right)}{4 \pi r_{i}^{2}} \times \sin |b|
\end{aligned}
$$



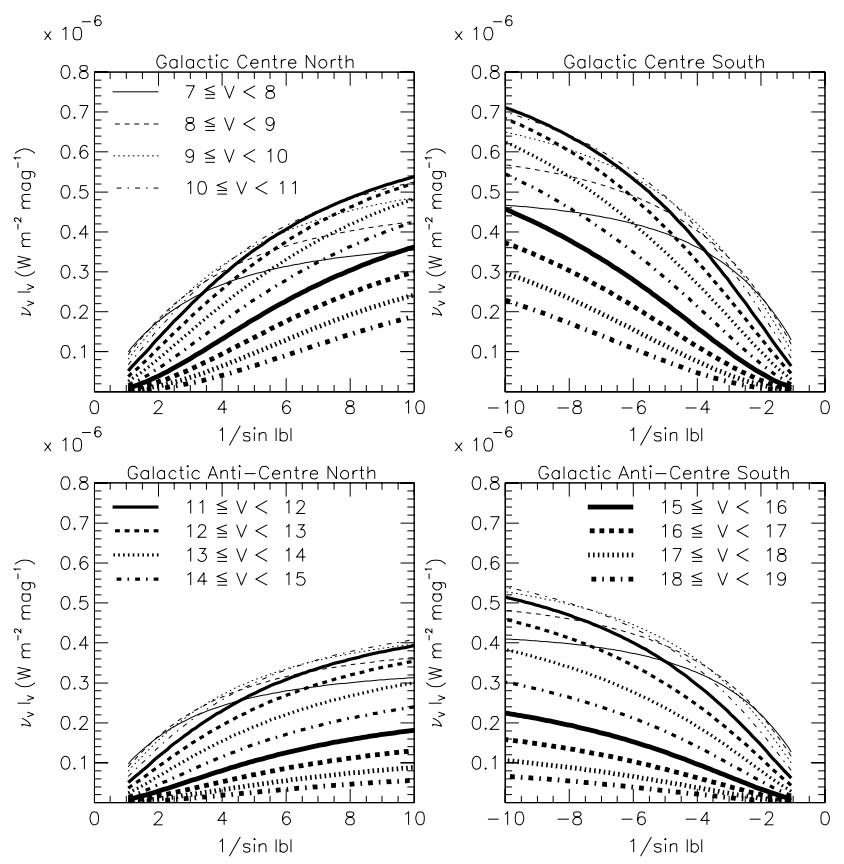

Fig. 7. Simulations in $V$ with extinction. Integrated $V$ flux in 1 mag bins as a function of $1 / \sin |b|$ for fainter stars $(V \geq 7)$.

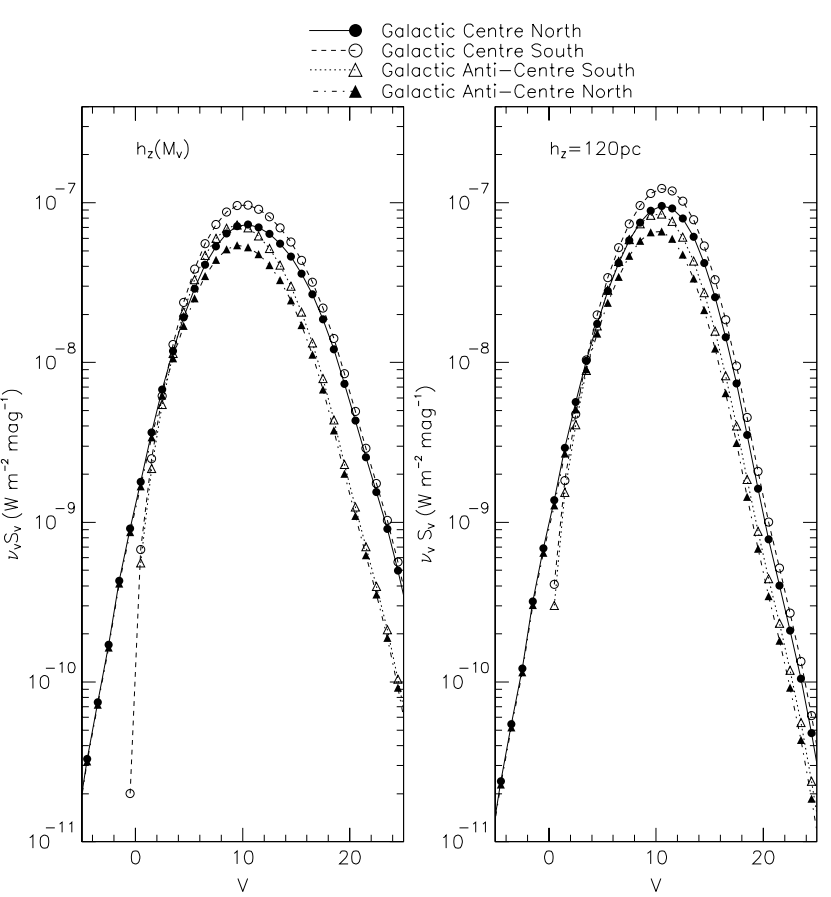

Fig. 8. Simulations in $V$ with extinction. Contribution of each apparent $V$ magnitude bin to the surface brightness for 4 sky areas. On the left (resp. right) panel, the stellar density (Eq. (1)) is computed with a variable (resp. fixed) scale height $h_{Z}\left(M_{V}\right)\left(\right.$ resp. $\left.h_{Z}=120 \mathrm{pc}\right)$.

where $F_{v}\left(M_{V}, S p T\right)$ (resp. $\left.L_{\lambda}\left(M_{V}, S p T\right)\right)$ is the flux (resp. luminosity) spectrum in $\mathrm{W} \mathrm{m}^{-2} \mathrm{~Hz}^{-1}$ (resp. W) corresponding to a star with a magnitude $M_{V}$ and a spectral type SpT, and $r_{i}$ the actual distance of this star.

According to our simulations (Table A.1), the cut in apparent magnitude $(V<7.3)$ limits our sensitivity to $17 \%$ of the total $V$ stellar surface brightness. We apply a volume correction relying on the knowledge of the Hipparcos parallaxes together with the well-defined completeness limit $(V<7.3)$ : each

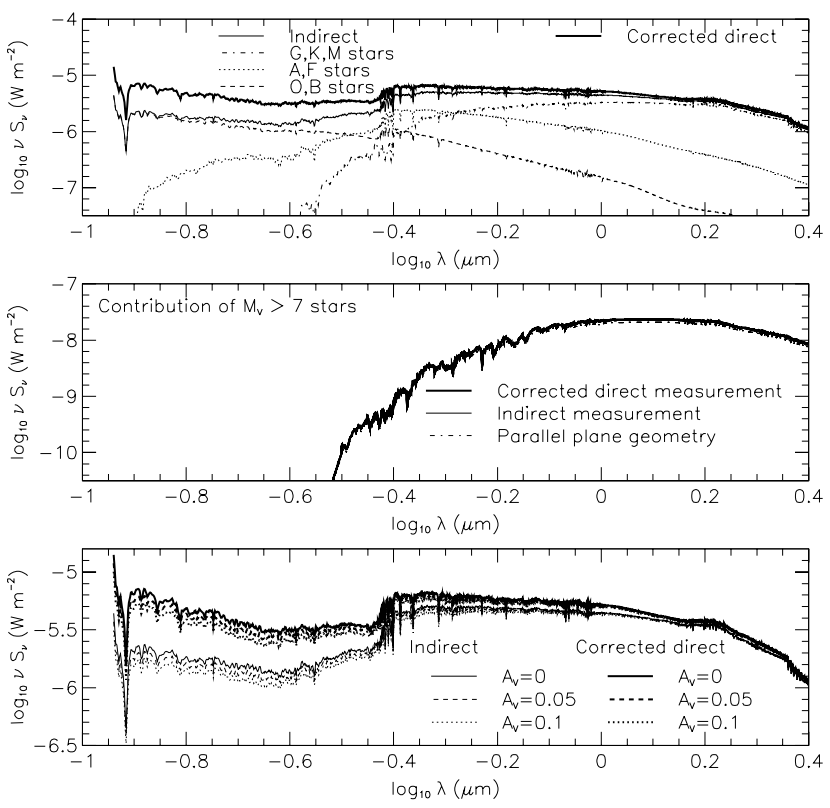

Fig. 9. Corrected direct and indirect synthetic estimates of the surface brightness of our Galaxy at the Solar Neighbourhood. In the top panel, we display the two estimates obtained with the compilation of the $V<7.3$ Hipparcos Catalogue stars. For the indirect estimate, we indicate the contribution of the different types of stars. In the middle panel, we display the contribution of $M_{V}>7$ stars with our simulation tool for different configurations. We also consider $\pm 1 \sigma$ luminosity functions to estimate the scatter due to uncertainties in the luminosity function. These dim stars mainly contribute to the near-infrared part of the spectrum, but this integrated surface brightness remains an order of magnitude below their bright counterparts. In the bottom panel, we study the influence of the extinction, perpendicular to the Galactic plane, which might have a marginal influence in the UV. We assume a fixed extinction $A_{V}$ applies to the whole population.

absolute magnitude bin $\left(M_{V}-\frac{1}{2} \leq m<M_{V}+\frac{1}{2}\right)$ is complete within a radius $R\left(M_{V}+\frac{1}{2}\right)$ (see Appendix D.2). We compute

$$
\begin{array}{r}
v S_{v}=2 \times \sum_{i}^{M_{V}<7} \sum_{\left[M_{V}-\frac{1}{2}, M_{V}+\frac{1}{2}[\right.}^{x_{i} \in} \frac{L_{\lambda}\left(x_{i}, S p T\right) \sin |b|}{4 \pi r^{2}} \times f_{i}^{\mathrm{LF}} \\
\times \frac{W_{*}^{\mathrm{sph}}\left(R_{\max }\right)}{W_{*}^{\mathrm{sph}}\left(R\left(x_{i}\right)\right)}
\end{array}
$$

here $L_{\lambda}\left(x_{i}, S p T\right)$ is the spectrum (in $\mathrm{W}$ ) corresponding to a star with a magnitude $M_{V}-\frac{1}{2} \leq x_{i}<M_{V}+\frac{1}{2}$ and a spectral type $\mathrm{SpT}, f_{i}^{\mathrm{LF}}$ is the correction of the luminosity function provided in Fig. D.4 applied for stars with $M_{V} \leq-1.5$, and $R_{\max }=1 \mathrm{kpc}$ is the cylinder radius discussed in Sect. 2 . The last factor corresponds to the volume correction with: $W_{*}^{\mathrm{sph}}(y)=\int_{r<y} n(r, b, l) \sin |b| / r^{2} \mathrm{~d} v, \mathrm{~d} v=\cos (b) \mathrm{d} b \mathrm{~d} l \mathrm{~d} r . W_{*}^{\mathrm{sph}}(y)$ corresponds to the stellar surface density weighted by $\sin |b| / r^{2}$ computed within a sphere of radius $\left.y . W_{*}^{\mathrm{sph}} R\left(x_{i}\right)\right)$ is computed for each magnitude bin, while $W_{*}^{\text {sph }}\left(R_{\max }\right)$ is obtained with a weighted sphere of radius equal to the cylinder radius $R_{\max }$. The corresponding spectrum is displayed in Fig. 9. We check that if we take $W_{*}^{\mathrm{sph}}\left(5 R_{\max }\right)$ instead of $W_{*}^{\mathrm{sph}}\left(R_{\max }\right)$, the estimate of the surface brightness increases by less than $14.5 \%$.

This measurement takes into account the real distribution of stars but assumes a smooth and homogeneous distribution of stars of the LF along the exponential profile of the Milky Way for the corrections. 


\subsection{Indirect measurement of starlight: a synthetic spectrum}

The second possibility is to compute the surface brightness as the mean stellar luminosity spectrum per unit area at the Solar Neighbourhood. We rely on Hipparcos data for intrinsically bright stars $\left(M_{V}<7\right)$ and perform the appropriate corrections, as follows:

$v I_{v}=\sum_{i}^{M_{V}<7} \sum_{\left[M_{V}-\frac{1}{2}, M_{V}+\frac{1}{2}[\right.}^{x_{i} \in} \frac{L_{\lambda}\left(x_{i}, S p T\right)}{\pi R\left(x_{i}\right)^{2}} \times f_{i}^{\mathrm{LF}} \times \frac{\Sigma_{i}^{\mathrm{cyl}}}{\Sigma_{i}^{\mathrm{sph}}}$

where $L_{\lambda}\left(M_{V}, S p T\right)$ is the spectrum (in W) corresponding to a star with a magnitude $M_{V}$ and a spectral type SpT, $f_{i}^{\mathrm{LF}}$ is the correction of the luminosity function. The last factor corresponds to the volume correction for each magnitude bin. $\Sigma_{i}^{\text {cyl }}$ is the stellar surface density computed for the cylinder of radius $R_{\max }$, while $\Sigma_{i}^{\text {sph }}$ is the stellar surface density integrated over a sphere of radius $R\left(x_{i}\right)$ normalised to the surface $\pi R\left(x_{i}\right)^{2}$.

This measurement assumes a smooth homogeneous distribution of the stars and a uniform LF along the exponential profile of the Milky Way.

\subsection{Discussion}

Figure 9 presents the corrected direct and indirect estimates. The two curves are very close in the optical and near-infrared, while they differ significantly in the UV. This difference, which cannot be explained by extinction (see bottom panel of Fig. 9), is understood as due to the inhomogeneous distribution of the OB associations (de Zeeuw et al. 1999, see also Appendix D.3). If we remove the very bright stars $\left(M_{V}<-4.5\right)$, the corrected direct estimate is reduced in the UV. If we remove the bright stars $\left(M_{V}<-1.5\right)$, all the discrepancies are removed.

The corrected direct estimate, based on the fluxes, is very sensitive to the anisotropic distribution of $\mathrm{OB}$ associations, while the indirect estimate is more robust, as it is based on luminosities, but does not account for the real spatial distribution of stars. The uncertainties on the bright end of the LF (see Fig. D.1) are also a source of error. By chance, our indirect estimate is in good agreement with the UV measurements of Gondhalekar et al. (1980) (see Fig. 16). However, it is a different quantity that has been measured: the sum of the UV light over the whole sky (S2/68 Sky-survey TD1-satellite data) without any extinction correction. This might mean that, due to an obvious selection bias, we detect only stars that are moderately extinguished, but this might also be a coincidence since the measured quantities are different.

The significant difference of our two estimates in the $\mathrm{UV}$ range is due to the irregular distribution of OB stars (considered homogeneous in the indirect estimate).

The surface brightness obtained in the optical can be compared with the study of Flynn et al. (2006). These authors use accurate data on the local luminosity function and the disc's vertical structure to measure the following surface brightnesses (see their Table 5): $\mu_{B}=23.51 \mathrm{mag} \operatorname{arcsec}^{-2}, \mu_{V}=$ $22.93 \mathrm{mag} \mathrm{arcsec}^{-2}$, and $\mu_{I}=22.03 \mathrm{mag} \operatorname{arcsec}^{-2}$. These values are in good agreement with our corrected direct estimates (see Tables 6 and 7). Our indirect estimates are slightly weaker for the reasons discussed above.

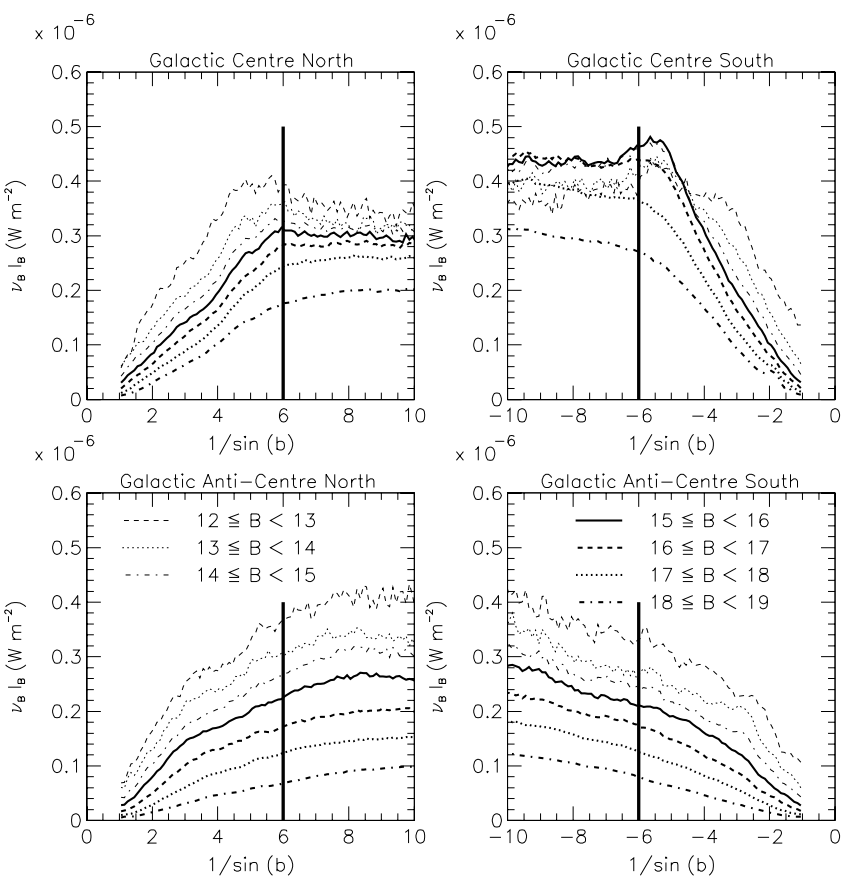

Fig. 10. Cosecant laws for $12 \leq B<19$ stars from the USNO-A2 catalogue. Irregularities, significant towards the Galactic centre, are minimised by our cut $1 / \sin |b|<6$ (see Table A.2).

\section{Optical surface brightness}

\subsection{USNO-A2 and TYCHO-2 data}

The USNO-A2 $B$ and $R$ magnitudes have been calibrated as explained in Appendix G.

\subsection{1. $B$ measurements}

Figures 10 and 11 display the cosecant laws obtained for 4 sky areas with the USNO-A2 and TYCHO-2 stellar catalogues and for each magnitude bin. Table A.2 summarises the slopes measured with the fit to the cosecant laws and provides a comparison with simulations. These simulations are based on the method described in Sect. 2.2. A $B$ luminosity function has been derived from the $V$ luminosity function displayed in Fig. D.1, assuming that $50 \%$ of the $V<2$ stars are red giants. We rely on Lang (1992, p. 137-145) to relate the $V$ absolute magnitudes to spectral types from Pickles (1998), and to perform this transformation. We thus find excellent agreement between our simulations and the TYCHO-2 data. The USNO-A2 data provide estimates systematically larger than our simulations. The emission in the Galactic Centre South direction is perturbed as displayed in Fig. 10. Note that simulations show that $B>19$ stars do not contribute more than a few percent to the total surface brightness.

We combine the $B$ USNO-A2 and TYCHO-2 estimates for $B>6$ stars with the synthetic uncorrected estimate based on $(B<6)$ Hipparcos stars (see Table 2 and Fig. 14), and correct the extinction with the global factor provided in Fig. 2 and Table E.1, as follows:

$$
\begin{aligned}
v_{B} S_{B} & =\left(5.640 \times 10^{-7}+3.004 \times 10^{-6}\right) \times 1.72 \\
& =6.13 \times 10^{-6} \mathrm{~W} \mathrm{~m}^{-2}=23.51 \mathrm{mag} \mathrm{arcsec}^{-2}
\end{aligned}
$$



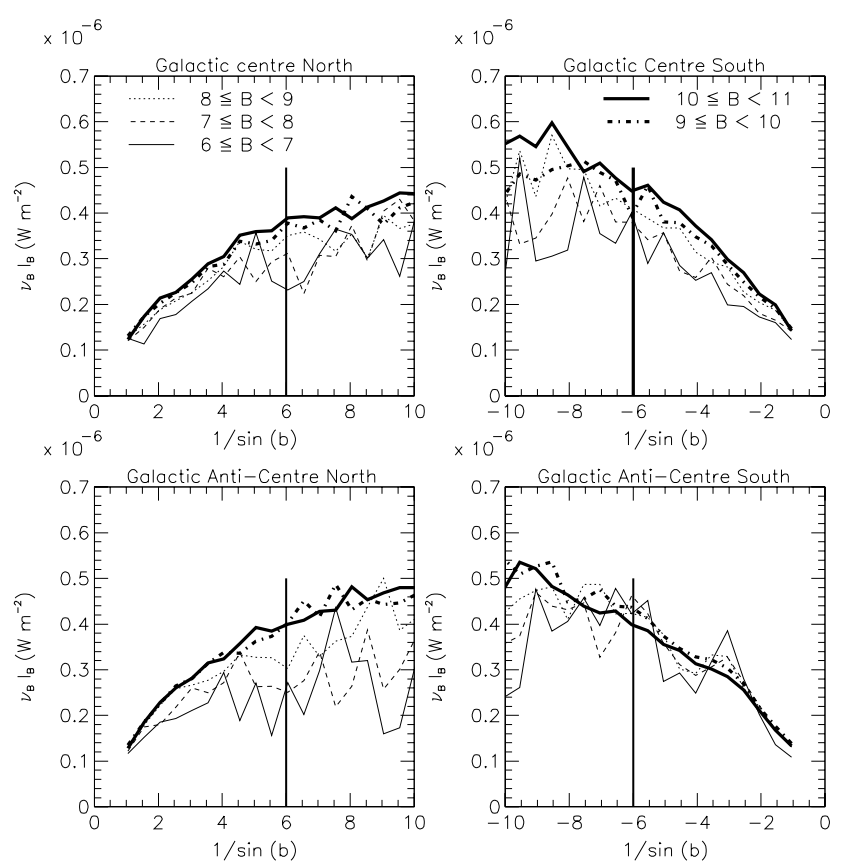

Fig. 11. Similar to Fig. 10 for $B$ cosecant laws computed for $6 \leq B<$ 11 TYCHO-2 stars (see Table A.2).

Table 2. Contribution of the $B<6$. stars to the surface brightness (see Fig. 14). 2783 Hipparcos stars have thus been used. Values with the observed extinction are provided together with the values corrected for extinction.

\begin{tabular}{rrr}
\hline \hline$\lambda(\mu \mathrm{m})$ & With extinction & Without extinction \\
\hline $0.440\left(B_{J}\right)$ & $5.640 \times 10^{-7}$ & $6.186 \times 10^{-7}$ \\
\hline
\end{tabular}

\subsection{2. $R$ measurements}

The data reduction of the $R$ measurements of the calibrated USNO-A2 data is presented in Table A.3 and Fig. 12. We clearly observe an excess of the stellar data with $12<R<18$ with respect to our simulation ${ }^{4}$. This cannot not be accounted for by the thick disc, which is dominated by dim stars $\left(M_{V}>4\right)$ that do not contribute significantly to the surface brightness (see Fig. D.2). We carefully check with the USNO-B1 (Monet et al. 2003) catalogue that our calibration is satisfactory. In addition, this excess has been confirmed with the CCD UCAC-1 data (see Appendix F).

We complete the $12 \leq R<18$ USNO-A2 estimate with simulations presented in Table A.3, and correct the extinction with the global factor provided in Fig. 2 and Table E.1, as follows:

$$
\begin{aligned}
v_{R} S_{R} & =\left(2.205 \times 10^{-6}+2.555 \times 10^{-6}\right) \times 1.41 \\
& =6.71 \times 10^{-6} \mathrm{~W} \mathrm{~m}^{-2}=22.6 \mathrm{mag} \mathrm{arcsec}^{-2} .
\end{aligned}
$$

\subsection{Pioneer $10 / 11$ data}

As discussed in Gordon et al. (1998) (see also Leinert et al. 1998), the Pioneer 10/11 data provide an excellent all-sky survey in $B(0.437 \mu \mathrm{m})$ and $R(0.644 \mu \mathrm{m})$ for the study of integrated

${ }^{4}$ To account for red giants, we apply in $R$ the modification of the luminosity function described in Sect. 4.1.1 to perform this simulation.
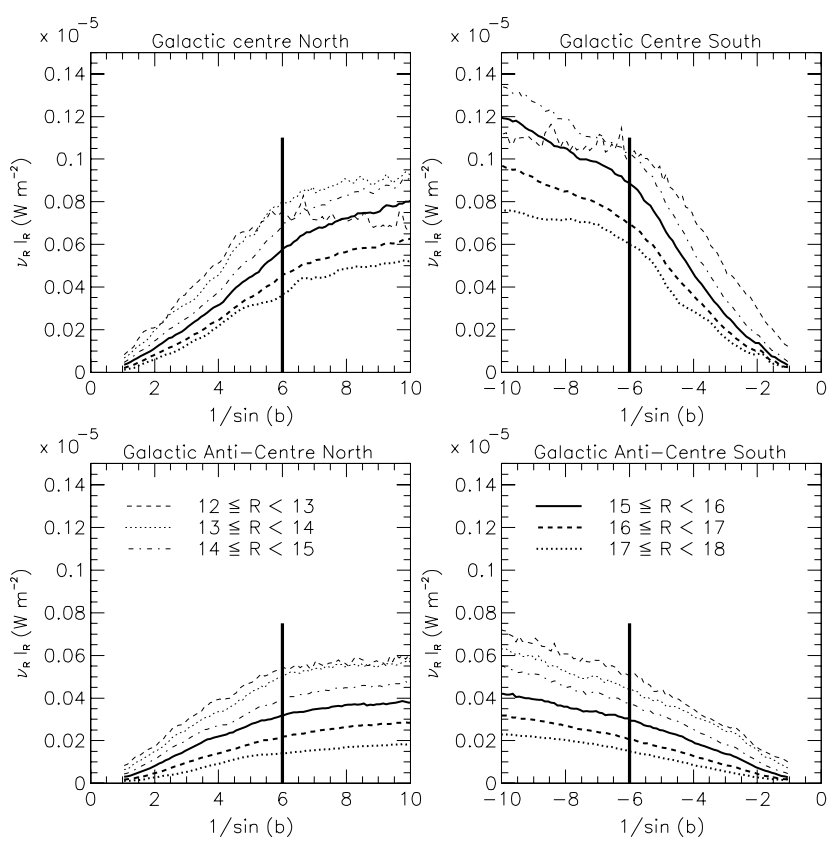

Fig. 12. Integrated $R$ fluxes as a function of $1 / \sin (b)$ computed with the USNO-A2 data for $12 \leq R<18$ stars. We decompose the contributions originating from different sky areas. No correction has been applied (see Table A.3).

Table 3. Contribution of the $R<6$. stars to the surface brightness computed the same way as those displayed in Fig. 14. 7010 Hipparcos stars are thus considered. Values with the observed extinction are provided together with the values corrected for extinction.

\begin{tabular}{rrr}
\hline \hline$\lambda(\mu \mathrm{m})$ & With extinction & Without extinction \\
\hline $0.640(R)$ & $6.98 \times 10^{-7}$ & $7.41 \times 10^{-7}$ \\
\hline
\end{tabular}

flux. In contrast to the Tycho star mapper background analysis of Wicenec \& van Leeuwen (1995), the Pioneer 10/11 data taken beyond 3.26 AU provide sky-maps devoid of detectable zodiacal light. We use here the Pioneer 10/11 maps kindly provided by K. Gordon (see Gordon et al. 1998 and Witt et al., in preparation): they correspond to the first iteration maps discussed in Gordon et al. (1998). These maps contain all relevant flux (starlight and possible diffuse components), except for stars brighter than $V=6.5$ (see Toller et al. 1987). Figure 13 displays the $1 / \sin (b)$ laws obtained in $B$ and $R$ for the different sky areas, while Table 4 provides the corresponding slopes. The $R$ fluxes are systematically larger than the $B$ fluxes: as discussed in the following, this is a consequence of the extinction.

Relying on Eq. (5), we compute the contribution of the bright $(V<6.5)$ stars, independently with a synthetic direct estimate based on the Hipparcos Catalogue, which is complete for these stars. We nevertheless neglect stars with uncertain parallax or undefined spectral type, but estimate that they do not contribute more than $10 \%$ in $V$.

We combine the Pioneer $B$ and $R$ estimates with the $(V<$ 6.5) stars synthetic uncorrected estimates, and correct the extinction with the global factor provided in Fig. 2 and Table E.1 (see also Appendix E), as follows:

$$
\begin{aligned}
S_{B}= & \left(7.02 \times 10^{-7}+4.52 \times 10^{-6}\right) \times 1.72 \\
& =8.98 \times 10^{-6} \mathrm{~W} \mathrm{~m}^{-2}=23.1 \mathrm{mag} \operatorname{arcsec}^{-2} \\
S_{R} & =\left(7.26 \times 10^{-7}+6.04 \times 10^{-6}\right) \times 1.41 \\
& =9.54 \times 10^{-6} \mathrm{~W} \mathrm{~m}^{-2}=22.3 \mathrm{mag} \operatorname{arcsec}^{-2}
\end{aligned}
$$


Table 4. $B(0.437 \mu \mathrm{m})$ and $R(0.644 \mu \mathrm{m})$ (Pioneer $10 / 11$ derived) surface brightness, given in $10^{-9} \mathrm{~W} \mathrm{~m}^{-2}$. We give the values fitted for $1 / \sin (b)<6$ (see Fig. 13). The surface brightness thus obtained accounts for the $V>6.5$ stars as well as for a possible diffuse component.

\begin{tabular}{|c|c|c|c|c|c|c|c|c|}
\hline \multirow{3}{*}{$\lambda(\mu \mathrm{m})$} & \multicolumn{4}{|c|}{ Galactic centre } & \multicolumn{4}{|c|}{ Galactic anti-centre } \\
\hline & \multicolumn{2}{|c|}{ North } & \multicolumn{2}{|c|}{ South } & \multicolumn{2}{|c|}{ North } & \multicolumn{2}{|c|}{ South } \\
\hline & $|b|=90^{\circ}$ & slope & $|b|=90^{\circ}$ & slope & $|b|=90^{\circ}$ & slope & $|b|=90^{\circ}$ & slope \\
\hline $0.437(B)$ & 1050. & 1148. & 1048. & 1444. & 1046. & 886. & 996. & 1040. \\
\hline $0.644(R)$ & 1368. & 1684. & 1542. & $\begin{array}{l}1958 . \\
\text { Sum }\end{array}$ & 1320. & 1164. & 1500. & 1234. \\
\hline $\begin{array}{l}0.437(B) \\
0.644(R)\end{array}$ & \multicolumn{4}{|c|}{$\begin{array}{l}\text { 2098. }\left(|b|=90^{\circ}\right), 2592 . \text { (slope) } \\
\text { 2910. }\left(|b|=90^{\circ}\right), 3642 . \text { (slope) }\end{array}$} & \multicolumn{4}{|c|}{ 2042. $\left(|b|=90^{\circ}\right), 1926$. (slope) } \\
\hline \multicolumn{9}{|c|}{$\begin{array}{l}\text { Total } B(\mathbf{0 . 4 3 7} \boldsymbol{\mu m}) \text { value: } 4140 .\left(|b|=90^{\circ}\right), 4518 . \text { (slope) } \\
\text { Total } \boldsymbol{R}(\mathbf{( 0 . 6 4 4} \boldsymbol{\mu} \mathbf{m}) \text { value: } 5730 .\left(|b|=90^{\circ}\right), 6040 \text { (slope) }\end{array}$} \\
\hline
\end{tabular}
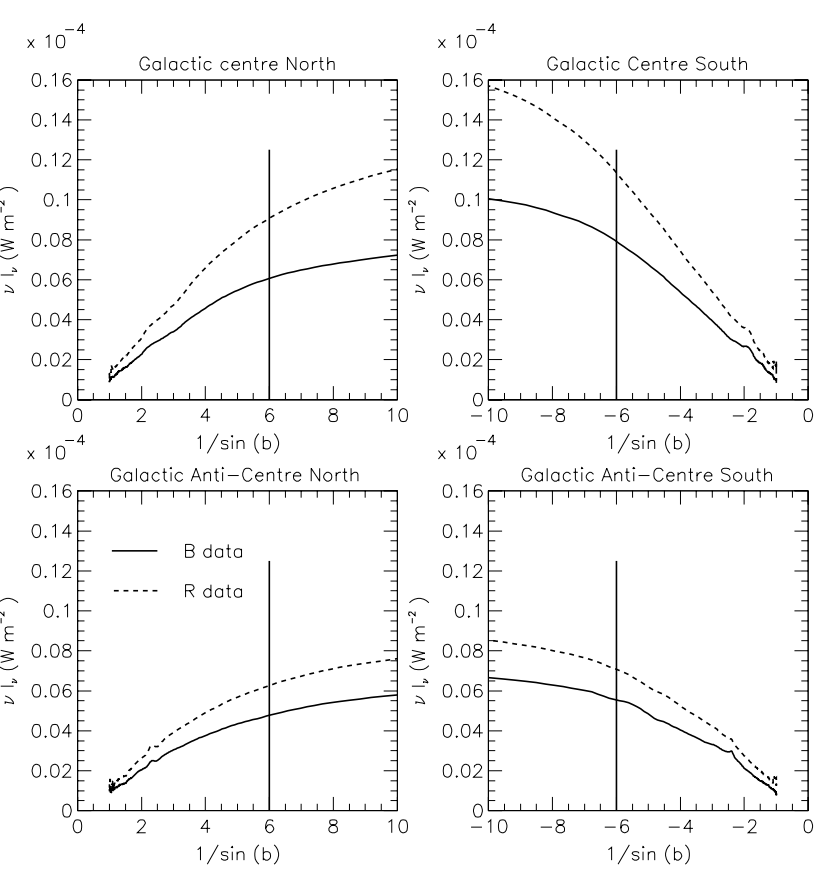

Fig. 13. Cosecant laws for Pioneer $10 / 11 B$ (full-line) and $R$ (dashedline) data (Gordon et al. 1998). This corresponds to $V>6.5$ stars and diffuse emission. There is a hole in the Galactic Anti-Centre North area (bottom left) that has been removed. No correction has been applied (see Table 4).

Our estimate is brighter than the estimate of the surface brightness obtained in $B$ from the Pioneer data $\left(\mu_{B}=23.8 \pm\right.$ $0.1 \mathrm{mag} \mathrm{arcsec}^{-2}$ ) by van der Kruit (1986), who did not correct for extinction.

The values obtained here are systematically brighter than our synthetic estimates and than the local values provided by Flynn et al. (2006). As will be discussed in Sect. 6, we suggest that this disagreement is due to the larger volume probed by the USNO-A2 and Pioneer 10/11 all-sky surveys.

\section{Infrared surface brightness: COBE/DIRBE data}

We consider the Zodi-Subtracted Mission Average (ZSMA) maps (Kelsall et al. 1998) from the COBE/DIRBE project. We plot the cosecant laws for the wavelengths 1.25, 2.2, 3.5, 4.9, 12, 25, 60, 100, 140 and $240 \mu \mathrm{m}$ as shown in Fig. 15 (for $1.25 \mu \mathrm{m}$ ). We then perform a linear fit (slope and $|b|=90^{\circ}$ values) with the parameters provided in Table A.4. We are sensitive to bright point sources (like Betelgeuse) up to $4.9 \mu \mathrm{m}$, which we eliminate with a $5 \sigma$ clip when necessary. Unlike Boulanger \& Pérault (1988), we do not subtract nearby dust complexes from the study

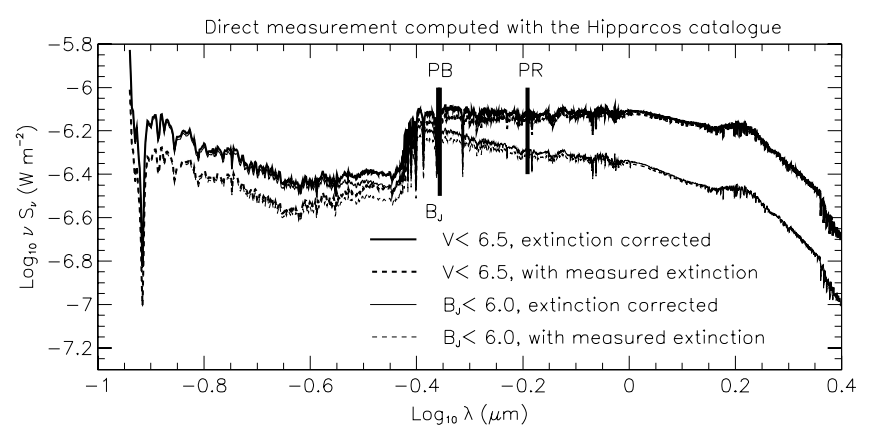

Fig. 14. Direct contribution of bright stars to the surface brightness. These synthetic estimates have been computed with Eq. (6) for $V<6.5$ stars and for $B<6$ stars from the Hipparcos catalogue. 8013 and 2783 stars have thus been used, while the few neglected stars do not contribute to more than $10 \%$ in $V$ (see Table 5). The vertical lines indicate the wavelengths discussed in the text $\left(B_{J}\right.$ and Pioneer $\left.B / R\right)$. Note that the extinction considered here is the extinction affecting each individual star not the global extinction perpendicular to the Galactic plane.
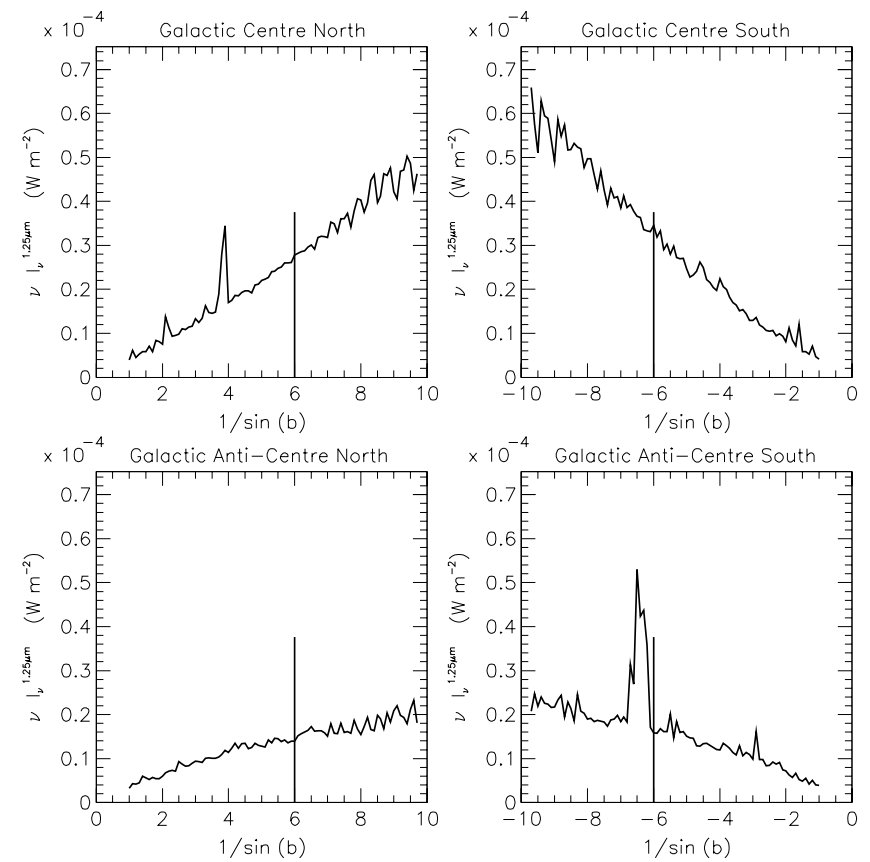

Fig. 15. Cosecant laws for the $1.25 \mu \mathrm{m}$ COBE/DIRBE data averaged over Galactic latitude. These values are derived from Zodi-Subtracted Mission Average (ZSMA) data distribution. The sharp peaks correspond to bright stars $(0<V<1$ and $\mathrm{M}$ type). We performed similar plots for the other $9 \mathrm{COBE} / \mathrm{DIRBE}$ available wavelengths. Wiggles observed for $1 / \sin |b|>6$ are due to the COBE/DIRBE sampling (pixels of $0.32^{\circ} \times 0.32^{\circ}$ ). 


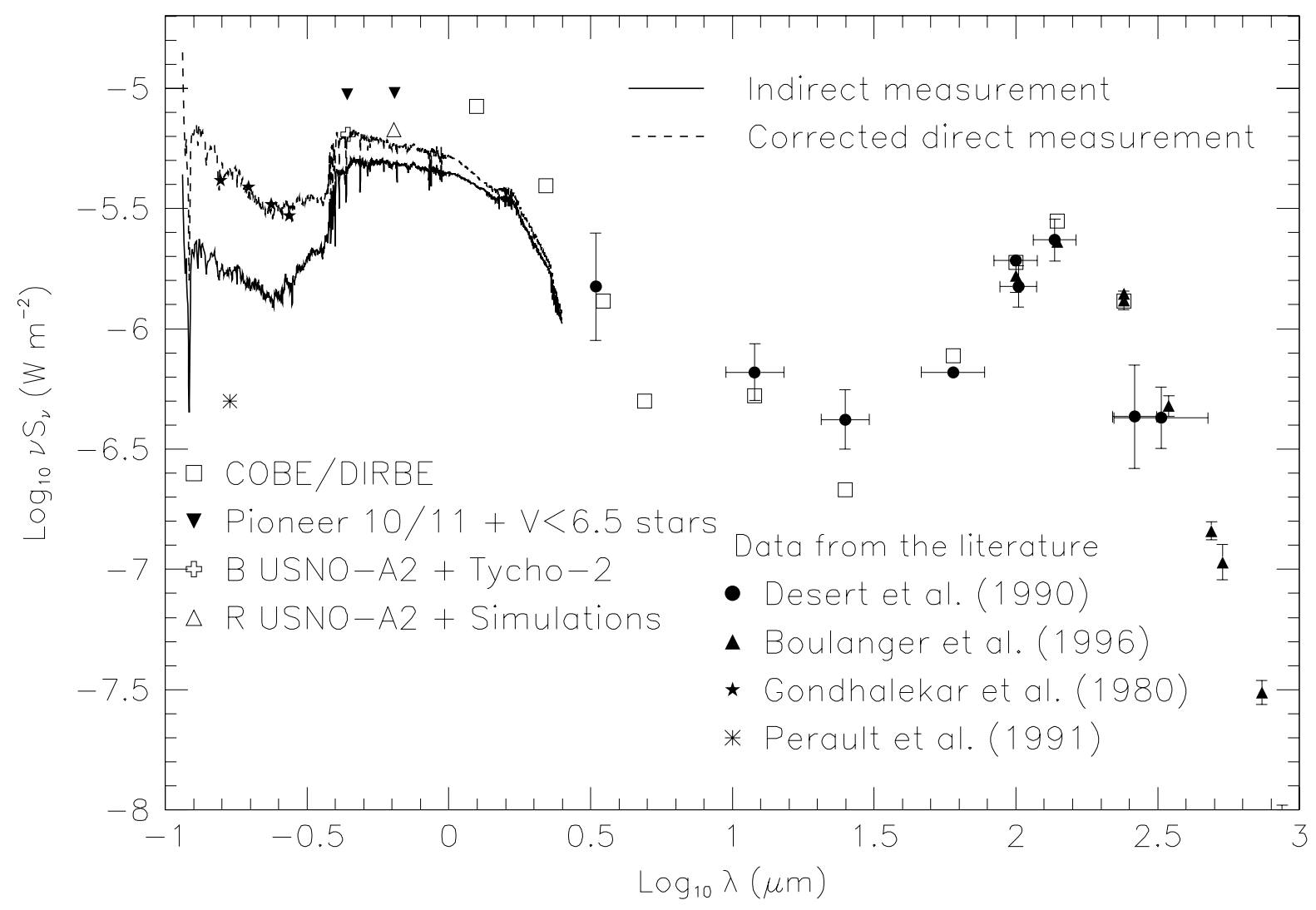

Fig. 16. Summary of the measurements discussed throughout this paper. The data points with no error bars have been computed with the cosecant law extrapolation method applied at given wavelengths (COBE/DIRBE, Pioneer 10/11, USNO-A2 and Tycho-2). The Pioneer 10/11 and COBE/DIRBE $1.25 \mu \mathrm{m}$ and $2.2 \mu \mathrm{m}$ are corrected for extinction (cf. Table E.1). In parallel, the dashed and full line curves correspond to the stellar synthetic estimates based on Hipparcos data. In the IR, our estimates based on COBE/DIRBE are compatible with Désert et al. (1990), Boulanger et al. (1996) (symbols with error bars). As discussed in Sect. 5, the $12 \mu \mathrm{m}$ and $25 \mu \mathrm{m}$ data points are probably affected by uncertainties due to Zodiacal light residuals. In the UV, the uncertainties are large due to the sensitivity to inhomogeneities (OB associations). Our indirect estimate is compatible with the all-sky average performed by Gondhalekar et al. (1980).

as we are interested in the light of all the components. Otherwise, we observe a quite linear behaviour except at 12 and $25 \mu \mathrm{m}$ for which the light emission is very irregular, as observed by Boulanger \& Pérault (1988) with IRAS data. These data exhibit a large value of the $|b|=90^{\circ}$ intensity and a small value of the slope. This large $|b|=90^{\circ}$ intensity is characteristic of a "nonGalactic" or at least irregular behaviour, and also seems to affect marginally the $4.90 \mu \mathrm{m}$ data. At 12 and $25 \mu \mathrm{m}$, we observe an unexplained excess for $1 / \sin |b|<2\left(b>30^{\circ}\right)$, which affects the fit. A strange behaviour was also noted by Boulanger \& Pérault (1988) in this area and interpreted as a consequence of imperfect Zodiacal light subtraction. Hence, we perform the fit for $2<1 / \sin |b|<6$ at these wavelengths in order to minimise the effect, but these measurements are probably still affected by noise and systematic effects. Note that Arendt et al. (1998), who studied the Galactic foreground from the same ZSMA data, have identified similar spurious effects and explained them as being residuals of the Zodiacal light subtraction.

For $\lambda<5 \mu \mathrm{m}$, we observe the expected asymmetry between the North and South hemispheres due to the position of the Sun above the Galactic plane: the integrated radiation intensity measured in the Galactic Center (resp. Anti-Centre) South panel is larger than the one measured in the Galactic Center (resp. AntiCentre) North panel. At longer wavelengths, we observe an inverted behaviour: the radiation intensity measured in Galactic North panels is larger than the one measured in the South panels. This is explained by the presence of a warp of the Galactic
Table 5. Contribution of the $V<6.5$ stars to the surface brightness (see Fig. 14). Values with the observed extinction are provided together with the values corrected for extinction. We estimate that we do not miss ${ }^{1}$ more than $3 \%$ of the brightness in $V$.

\begin{tabular}{rrr}
\hline \hline$\lambda(\mu \mathrm{m})$ & With extinction & Without extinction \\
\hline $0.437(B)$ & $7.020 \times 10^{-7}$ & $7.751 \times 10^{-7}$ \\
$0.644(R)$ & $7.261 \times 10^{-7}$ & $7.699 \times 10^{-7}$ \\
$V$ bad stars ${ }^{1}$ & $2.465 \times 10^{-8}$ & \\
\hline
\end{tabular}

${ }^{1}$ This corresponds to stars with uncertain parallax or unspecified spectral type, which were neglected for the previous direct estimates.

disc (beyond $12 \mathrm{kpc}$ ) towards the North and Galactic Centre (see e.g. Burton \& Te Lintel Hekkert 1986; Wouterloot et al. 1990). Last, for $\lambda \geq 60 \mu \mathrm{m}$, we are affected by nearby complexes (that we choose not to remove): this tends to enlarge the uncertainties but accounts for local fluxes. This probably explains in part the relatively low values of the intercepts $\left(|b|=90^{\circ}\right)$ found for those wavelengths. However, as further discussed in the next section, and displayed in Fig. 16, the values of the slopes thus obtained are fully compatible with those used by Désert et al. (1990) based in part on IRAS data, and those computed by Boulanger et al. (1996) based on COBE/DIRBE and FIRAS data. 
Table 6. Summary of surface brightness measurements for the usual optical bands and comparison with other estimates. The various estimates discussed in this paper have been gathered and provided in the different usual units. As discussed in the text, the values obtained for Pioneer 10/11, probing a larger volume than the (corrected) synthetic estimates based on the Hipparcos data, are systematically larger (brighter). Our (corrected direct) synthetic estimate is compatible with the Flynn et al. (2006) data in $B$ and $V$. In the $I$ band, the Flynn et al. (2006) estimate is between our synthetic and Pioneer 10/11 estimates. This could be a bias of our incompleteness correction, used for synthetic estimates, based on $V$ data, which assumes homogeneity of the stellar distribution. However, the Pioneer data (supported by the COBE/DIRBE near-infrared) estimates suggest that the surface brightness of the Milky Way is brighter. This could explain why Flynn et al. (2006) observed that the Milky Way appears to be underluminous with respect to the main locus of the Tully-Fisher relation for external galaxies.

\begin{tabular}{|c|c|c|c|c|c|c|c|c|}
\hline & Unit & $\bar{U}$ & $\bar{B}$ & $\bar{V}$ & $R_{C}$ & $R_{J}$ & $\overline{I_{C}}$ & $\bar{I} I_{J}$ \\
\hline$\lambda$ & $\dot{A}$ & 3660 & 4400 & 5530 & 6400 & 6930 & 7900 & 8785 \\
\hline$c_{\lambda}$ & $\mathrm{W} \mathrm{m}^{-2}$ & $1.48 \times 10^{-8}$ & $2.90 \times 10^{-8}$ & $1.97 \times 10^{-8}$ & $1.44 \times 10^{-8}$ & $1.25 \times 10^{-8}$ & $9.66 \times 10^{-9}$ & $7.77 \times 10^{-9}$ \\
\hline$L_{\odot}^{\lambda}=\lambda F_{\odot}^{\lambda}$ & $L_{\odot}$ & 0.282 & 0.558 & 0.745 & 0.753 & 0.737 & 0.661 & 0.622 \\
\hline \multicolumn{9}{|c|}{ This paper } \\
\hline \\
\hline \multirow[t]{4}{*}{ - corrected direct } & $10^{-6} \mathrm{~W} \mathrm{~m}^{-2}$ & 3.662 & 6.249 & 6.081 & 5.848 & 5.758 & 5.46 & 5.347 \\
\hline & $L_{\odot} \mathrm{pc}^{-2}$ & 9.08 & 15.49 & 15.07 & 14.50 & 14.27 & 13.56 & 13.25 \\
\hline & $L_{\odot}^{\lambda} \mathrm{pc}^{-2}$ & 32.20 & 27.76 & 20.23 & 19.26 & 19.36 & 20.51 & 21.30 \\
\hline & mag/" & 23.34 & 23.49 & 23.10 & 22.80 & 22.66 & 22.44 & 22.23 \\
\hline \multirow[t]{4}{*}{ - indirect } & $10^{-6} \mathrm{~W} \mathrm{~m}^{-2}$ & 2.433 & 4.618 & 4.924 & 4.864 & 4.817 & 4.597 & 4.495 \\
\hline & $L_{\odot} \mathrm{pc}^{-2}$ & 6.03 & 11.45 & 12.21 & 12.06 & 11.94 & 11.40 & 11.14 \\
\hline & $L_{\odot}^{\lambda} \mathrm{pc}^{-2}$ & 21.38 & 20.52 & 16.39 & 16.36 & 16.20 & 17.25 & 17.91 \\
\hline & mag/" & 23.78 & 23.81 & 23.33 & 23.00 & 22.86 & 22.63 & 22.41 \\
\hline \multirow[t]{4}{*}{ USNO-A2 } & $10^{-6} \mathrm{~W} \mathrm{~m}^{-2}$ & & 6.13 & & 6.71 & & & \\
\hline & $L_{\odot} \mathrm{pc}^{-2}$ & & 15.19 & & 16.63 & & & \\
\hline & $L_{\odot}^{\lambda} \mathrm{pc}^{-2}$ & & 27.23 & & 22.08 & & & \\
\hline & mag/" & & 23.51 & & 22.6 & & & \\
\hline \multirow[t]{4}{*}{ Pioneer $10 / 11^{a}$} & $10^{-6} \mathrm{~W} \mathrm{~m}^{-2}$ & $(6.23)$ & 8.98 & $(10.34)$ & 9.54 & $(9.79)$ & $(9.30)$ & $(9.51)$ \\
\hline & $L_{\odot} \mathrm{pc}^{-2}$ & (15.44) & 22.26 & $(25.63)$ & 23.65 & $(24.27)$ & $(23.05)$ & $(23.58)$ \\
\hline & $L_{\odot}^{\lambda} \mathrm{pc}^{-2}$ & $(54.76)$ & 39.89 & $(34.41)$ & $(31.41)$ & $(32.93)$ & (34.87) & $(37.90)$ \\
\hline & mag/" & $(22.76)$ & 23.09 & $(22.52)$ & 22.27 & $(22.09)$ & $(21.86)$ & $(21.60)$ \\
\hline \multirow[t]{4}{*}{ Basic simulations } & $10^{-6} \mathrm{~W} \mathrm{~m}^{-2}$ & & 5.13 & 4.23 & 4.76 & & & \\
\hline & $L_{\odot} \mathrm{pc}^{-2}$ & & 12.72 & 10.51 & 11.81 & & & \\
\hline & $L_{\odot}^{\lambda} \mathrm{pc}^{-2}$ & & 22.79 & 14.11 & 15.68 & & & \\
\hline & mag/" & & 23.67 & 23.49 & 23.02 & & & \\
\hline \multirow{5}{*}{ v. der Kruit (1986) } & & $\mathrm{Co}$ & parison with & her works ${ }^{b}$ & & & & \\
\hline & $10^{-6} \mathrm{~W} \mathrm{~m}^{-2}$ & & 4.68 & & & & & \\
\hline & $L_{\odot} \mathrm{pc}^{-2}$ & & 11.61 & & & & & \\
\hline & $L_{\odot}^{\lambda} \mathrm{pc}^{-2}$ & & 20.81 & & & & & \\
\hline & mag/" & & 23.8 & & & & & \\
\hline \multirow[t]{4}{*}{ Flynn et al. (2006) } & $10^{-6} \mathrm{~W} \mathrm{~m}^{-2}$ & & 6.12 & 7.09 & & & 7.96 & \\
\hline & $L_{\odot} \mathrm{pc}^{-2}$ & & 15.16 & 17.57 & & & 19.74 & \\
\hline & $L_{\odot}^{\lambda} \mathrm{pc}^{-2}$ & & 27.70 & 23.59 & & & 29.86 & \\
\hline & mag/" & & 23.51 & 22.93 & & & 22.03 & \\
\hline
\end{tabular}

${ }^{a}$ Values provided in parenthesis have been obtained as an interpolation based on the corrected direct template adjusted on the Pioneer $10 / 11$ and COBE/DIRBE measurements (factor 1.7).

${ }^{b}$ The surface brightness measurements in $\mathrm{W} \mathrm{m}^{-2}, L_{\odot} \mathrm{pc}^{-2}$ and $L_{\odot}^{\lambda} \mathrm{pc}^{-2}$ provided for the estimates of van der Kruit (1986) and Flynn et al. (2006) have been derived with the calibration constants $c_{\lambda}$ and $L_{\odot}^{\lambda}$ provided at the top of this table and computed with Eq. (B.2). These $L_{\odot}^{\lambda}$ pc ${ }^{-2}$ values are compatible with the original values within $10 \%$ uncertainties, due to the difference of $L_{\odot}^{\lambda}$ solar luminosity considered.

Table 7. Summary of surface brightness measurement in the near-infrared and in the infrared comparison with previous measurements. Our measurements are compatible with those of Désert et al. (1990). Both are systematically larger than our synthetic estimates based on the $V$ luminosity function, corrected for incompleteness assuming a homogeneous stellar density.

\begin{tabular}{|c|c|c|c|c|c|c|c|c|c|c|c|}
\hline$\lambda$ & $\begin{array}{l}\text { Unit } \\
\mu \mathrm{m}\end{array}$ & $\begin{array}{c}J \\
1.25\end{array}$ & $\begin{array}{c}K \\
2.20\end{array}$ & $\begin{array}{c}L \\
3.50\end{array}$ & 4.90 & 12 & 25 & 60 & 100 & 140 & 240 \\
\hline \multicolumn{12}{|c|}{ This paper } \\
\hline DIRBE & $10^{-6} \mathrm{~W} \mathrm{~m}^{-2}$ & 7.50 & 3.74 & 1.31 & 0.50 & 0.53 & 0.21 & 0.77 & 1.88 & 2.81 & 1.30 \\
\hline Synthetic estimates & & & & & & & & & & & \\
\hline - corrected direct & $10^{-6} \mathrm{~W} \mathrm{~m}^{-2}$ & 4.30 & 2.00 & & & & & & & & \\
\hline - indirect & $10^{-6} \mathrm{~W} \mathrm{~m}^{-2}$ & 3.92 & 1.85 & & & & & & & & \\
\hline
\end{tabular}

$3.3 \mu \mathrm{m}$

\begin{tabular}{lllllll}
\hline Désert et al. $(1990)^{a}$ & $10^{-6} \mathrm{~W} \mathrm{~m}^{-2}$ & $1.5 \pm 0.8$ & $0.66 \pm 0.18$ & $0.42 \pm 0.12$ & $0.66 \pm 0.04$ & $1.92 \pm 0.07$
\end{tabular}

${ }^{a}$ We assume a column density of hydrogen $N_{\mathrm{H}}=3 \times 10^{20} \mathrm{~cm}^{-2}$. 


\section{Discussion}

On the one hand, Fig. 16 provides a summary of all the measurements performed in this paper. We also superpose data from the literature as indicated. Pérault et al. (1991) provide a cosecant measurement in the UV of the diffuse component. Interestingly, they confirm that this does not make a substantial contribution. On the other hand, Table 6 gives numerical values of the surface brightness for $U B V R I$ filters and a comparison with other works. Table 7 summarises similarly the values for near-infrared and infrared.

We have shown with simulations that the surface brightness of the Galaxy at the Solar Neighbourhood as observed from outside the Galaxy can be computed with a cosecant averaging of all-sky surveys. While face-on estimates performed on external galaxies are not very sensitive to extinction, our measurements severely suffer from extinction due to our internal view point. We have computed the extinction corrections necessary to apply to the direct cosecant averaging estimates. Relying on these results, we compute synthetic spectral estimates of the optical surface brightness. We use the Hipparcos catalogue and the Hipparcos Input Catalogue together with a stellar spectral flux library to estimate the expected contribution of stars in the optical. We use knowledge of the stellar parallaxes and of the completeness limit to perform this estimate. We attempt to correct the incompleteness (uncertain parallaxes and stars below the completion limits) with corrections based on our simulations. We then compile optical and infra-red sky-surveys to obtain direct observational estimates.

We observe a systematic difference between our synthetic estimates and the optical and infra-red sky-surveys. The estimate based on USNO-A2 $R$ flux in the magnitude range $12 \leq R<18$ exhibits a large excess with respect to our simulations. This excess is compatible with the disagreement observed between Pioneer 10/11 and our synthetic estimates. On the other hand, in the $B$ band the TYCHO and USNO-A2 data are in relatively good agreement with the simulations and with our synthetic estimate. The Pioneer 10/11 estimate nevertheless exhibits an excess ( similar to what is observed in $R$ ). Various arguments could be invoked to provide tentative explanations for these differences:

- The USNO-A2 data exhibit a very irregular pattern in $B$, which is not observed in $R$. This might be an indication of patchy absorption affecting solely the $B$ data. Patchy extinction is not included in our simulations nor in the extinction correction. However, we would expect the Pioneer 10/11 data to be affected the same way. There might be some other not understood problem with the $B$ data.

- The synthetic estimates are based on the Hipparcos data only (distance limited sample with $V<7.3$ ), while the all-sky surveys integrate the whole sky. In practice, the all-sky surveys probe an equivalent cylinder radius of $1 \mathrm{kpc}$, while the synthetic estimates are based on the Hipparcos data, which is complete within $\sim 100-200 \mathrm{pc}$ depending on the magnitude range. In order to provide a surface brightness estimate within $1 \mathrm{kpc}$, we have applied appropriate corrections. However, these corrections are normalised to the local stellar density measured by Hipparcos and assume a uniform stellar density following the exponential disc geometry.

We suggest that this disagreement could be a signature of the presence of the local minimum of the stellar density linked with Gould's belt. As displayed in Fig. D.3, the magnitude interval $10 \leq V<18$ is roughly composed of $\left\langle M_{V}\right\rangle=4-5$ stars. In addition, we know that the strongest contribution comes from $-3<M_{V}<6$ (see Fig. D.2). The irregular distribution of $\mathrm{OB}$ stars (not accounted for in our correction for synthetic estimates, nor in our modelling) plays an important role.

- The all-sky surveys estimates follow the same profile as the synthetic estimates. This is also compatible with a minimum of the stellar density (the bright stars are the main contributors to the surface brightness).

- The Pioneer 10/11 data points, when uncorrected for extinction, are in good agreement with our synthetic estimate. However, they seem at odds with respect to the DIRBE/COBE $J$ and $K$ data. This is difficult to understand as the synthetic estimates are corrected for extinction (star by star).

The recent work of Flynn et al. (2006) based on (partly) different data sets and a careful modelling exhibits similar trends. Their Tuorla study (which results are quoted in Table 6) probes stars out to circa $200 \mathrm{pc}$. Their $B$ and $V$ estimates are consistent with our corrected direct synthetic estimates, since the volume probed with the Hipparcos data is similar. In parallel, their $I$-band estimate is systematically larger than our synthetic estimate and closer to our Pioneer 10/11 estimates. This is in agreement with our Gould's belt interpretation as I-band surface brightness should be less sensitive to inhomogeneities, and suggests that our incompleteness correction, assuming homogeneity of the stellar distribution (see Eq. (7)), probably misses some stars in I. Our results could explain why Flynn et al. (2006) find that the global luminosity of the Milky Way appears to be underluminous with respect to the main locus of the Tully-Fisher relation as observed for external galaxies.

We thus conclude that the presence of Gould's belt affects our measurements of the surface brightness of the Galaxy at the Solar Neighbourdhood. All-sky surveys (Pioneer 10/11 and DIRBE/COBE) do not suffer from magnitude limits and enable to probe the whole volume expected. Our synthetic estimates based on Hipparcos data underestimate the surface brightness by a factor about 2 . We tentatively interpret this difference as a signature of Gould's belt. The stellar catalogues tend partially to support this interpretation. In the $R$ band, we observed an excess in the USNO-A2 data compatible with the Pioneer 10/11 estimate.

Acknowledgements. We thank G. Lagache for her efficient help for the COBE/DIRBE data handling, R. Drimmel for providing an adapted version of his published J, K model, and A. Robin for providing us simulations prior to their publication. We are most grateful to $\mathrm{K}$. Gordon and his collaborators for providing their Pioneer 10/11 data, which have been very valuable for this study. We thank Guy Simon for preparing an adapted catalogue from the DENIS database. We also acknowledge useful discussions with J. Guibert and M. Pérault at various stages of this work. Work by A.G. was supported by grant AST 0452758 from the US NSF. A.L.M. has been supported during part of this work by a grant from the Société de Secours des Amis des Sciences. This research has made use of the NASA/IPAC Extragalactic Database (NED), which is operated by the Jet Propulsion Laboratory, California Institute of Technology, under contract with the National Aeronautics and Space Administration.

\section{References}

Arendt, R. G., Odegard, N. Weiland, J. L., et al. 1998, ApJ, 508, 74 [arXiv:astro-ph/9805323]

Boulanger, F., \& Pérault, M. 1988, A\&A, 330, 964

Boulanger, F., Abergel, A., Bernard, J.-P., et al. 1996, A\&A, 312, 256

Bucciarelli, B., García Yus, J., Casalegno, R., et al. 2001, A\&A, 368, 335

Burton, W. B., \& Te Lintel Hekkert, P. 1986, A\&AS, 65, 427

Dale, D. A., Helou, G., Contursi, A., Silbermann, N. A., \& Kolhatkar, S. 2001, ApJ, 549, 215

Désert, F., Boulanger, F., \& Puget, J.-L. 1990, A\&A, 237, 215

de Zeeuw, P. T., Hoogerwerf, R., de Bruijne, J. H. J., Brown, A. G. A., \& Blaauw, A. 1999, AJ, 117,354 
Drimmel, R., Cabrera-Lavers, A., \& López-Corredoira, M. 2003, A\&A, 409, 205 Fitzpatrick, E. L. 1999, PASP, 111, 63

Fouqué, P., Chevallier, L., Cohen, M., et al. 2000, A\&AS, 141, 313

Flynn, C., Holmberg, J., Protinari, L., Fuchs, B., \& Jahreiss, H. 2006, MNRAS, 372,1149

Gondhalekar, P. M., Philips, A., \& Wilson, R. 1980, A\&A, 85, 272

Gordon, K., Witt, A. N., \& Friedmann, B. C. 1998, ApJ, 498, 540

Guiderdoni, B., Hivon, E., Bouchet, F. R., \& Maffei, B. 1998, MNRAS, 295, 877

Hakkila, J., Myers, J. M., Stidham, B. J., \& Hartmann, D. H. 1997, AJ, 114, 2043

Humphreys, R., \& Larsen, J. Q. 1995, AJ, 110, 2183

Jahreiss, H., \& Wielen, R. 1997, in HIPPARCOS '97. Presentation of the Hipparcos and Tycho catalogs and first astrophysical results of the Hipparcos space astrometry mission, ed. B. Battrick, M. A. C. Perryman, \& P. L. Bernacca, 675

Kelsall, T., Weiland, J. L., Franz, B. A., et al. 1998, ApJ, 508, 44

Lallement, R., Welsh, B. Y., Vergely, J. L., Crifo, F., \& Sfeir, D. 2003, A\&A, 411,447

Lang, K. 1992, Astrophysical Data: Planets and Stars (New York: SpringerVerlag)

Leinert, C., Bowyer, S., Haikala, L. K., et al. 1998, A\&AS, 127, 1

Mathis, J. S., Mezger, P. G., \& Panagia, N. 1983, A\&A, 128, 212

Mattila, K. 1980a, A\&A, 82, 373
Mattila, K. 1980b, A\&AS, 39, 53

Mezger, P. G., Mathis, J. S., \& Panagia, N. 1982, A\&A, 105, 372

Miller, G. E., \& Scalo, J. M. 1979, ApJS, 41, 513

Monet, D. G., Levine, S. E., Canzian, B., et al. 2003, AJ, 125, 984

Monet, D., et al. 1998, USNO-A V2.0, A Catalog of Astrometric Standards, US Naval Observatory Flagstaff Station (USNOFS) and Universities Space Research Association (USRA) stationed at USNOFS

Pérault, M., Lequeux, J., Hanus, M., \& Joubert, M. 1991, A\&A, 246, 243

Perryman, M., Lindegren, L., Kovalevsky, J., et al. 1997, A\&A, 323, L49 Pickles, A. J. 1998, PASP, 110, 863

Poppel, W. 1997, Fund. Cosm. Phys., 18, 1

Robin, A. C., Crézé, M., \& Mohan, V. 1992, A\&A, 265, 32

Schlegel, D. J., Finkbeiner, D. P., \& Davis, M. 1998, ApJ, 500, 525

Toller, G., Tanabe, H., \& Weinberg, J.-L. 1987, A\&A, 188, 24

Turon, C., Gomez, A., Crifo, F., et al. 1992, A\&A, 258, 74

Turon, C., Requieme, Y., Grenon, M., et al. 1995, A\&A, 304, 82

van der Kruit, P. C. 1986, A\&A, 157, 230

van Leeuwen, F., Evans, D. W., Grenon, M., et al. 1997, A\&A, 323, 61

Wicenec, A., \& van Leeuwen, F. 1995, A\&A, 304, 160

Wouterloot, J. G. A., Brand, J., Burton, W. B., \& Kwee, K. K. 1990, A\&A, 230 , 21

Zacharias, N., Urban, S. E., Zacharias, M. I., et al. 2000, AJ, 120, 2131 
A.-L. Melchior et al.: The surface brightness of the Galaxy at the solar neighbourhood, Online Material $p 1$

\section{Online Material}


A.-L. Melchior et al.: The surface brightness of the Galaxy at the solar neighbourhood, Online Material $p 2$

\section{Appendix A: Tables}

Table A.1 is discussed in Sect. 2.1.3, and corresponds to Figs. 6 and 7. Table A.2 is discussed in Sect. 4.1.1 and corresponds to Figs. 10 and 11. Table A.3 is discussed in Sect. 4.1.2 and corresponds to Fig. 12. Table A.4 is discussed in Sect. 5. 
A.-L. Melchior et al.: The surface brightness of the Galaxy at the solar neighbourhood, Online Material p 3

Table A.1. Simulated $V$ surface brightness (in $10^{-9} \mathrm{~W} \mathrm{~m}^{-2}$ ) contributions obtained for each magnitude bin and sky area derived from the cosecant laws (see Figs. 6 and 7). We give the values fitted for $1 / \sin |b|<6$. The last column provides the percentages of the total surface brightness (PTSB) corresponding to each apparent magnitude bin.

\begin{tabular}{|c|c|c|c|c|c|c|c|c|c|}
\hline \multirow{3}{*}{ Mag. } & \multicolumn{4}{|c|}{ Galactic centre } & \multicolumn{4}{|c|}{ 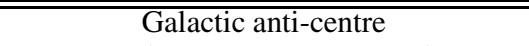 } & \multirow{3}{*}{$\begin{array}{r}\overline{P T S B} \\
\%\end{array}$} \\
\hline & \multicolumn{2}{|c|}{ North } & \multicolumn{2}{|c|}{ South } & \multicolumn{2}{|c|}{ North } & \multicolumn{2}{|c|}{ South } & \\
\hline & $\left(|b|=90^{\circ}\right)$ & slope & $|b|=90^{\circ}$ & slope & $|b|=90^{\circ}$ & slope & $|b|=90^{\circ}$ & slope & \\
\hline$-6 . \leq V<-5$ & 1.59 & 0.01 & 1.75 & -0.02 & 1.58 & 0.01 & 1.74 & -0.02 & 0.00 \\
\hline$-5 . \leq V<-4$ & 3.22 & 0.03 & 3.63 & -0.04 & 3.21 & 0.03 & 3.62 & -0.05 & 0.00 \\
\hline$-4 . \leq V<-3$. & 6.42 & 0.08 & 7.42 & -0.10 & 6.40 & 0.08 & 7.40 & -0.10 & 0.00 \\
\hline$-3 . \leq V<-2$ & 12.38 & 0.19 & 14.58 & -0.18 & 12.33 & 0.18 & 14.51 & -0.19 & 0.00 \\
\hline$-2 . \leq V<-1$. & 22.03 & 0.45 & 26.39 & -0.18 & 21.92 & 0.43 & 26.23 & -0.20 & 0.02 \\
\hline$-1 . \leq V<0$. & 33.65 & 0.95 & 41.28 & 0.04 & 33.41 & 0.90 & 40.95 & -0.02 & 0.07 \\
\hline $0 . \leq V<1$ & 46.21 & 1.92 & 58.39 & 0.83 & 45.73 & 1.80 & 57.72 & 0.69 & 0.19 \\
\hline 1. $\leq V<2$. & 60.78 & 3.81 & 79.89 & 2.68 & 59.90 & 3.53 & 78.65 & 2.33 & 0.44 \\
\hline 2. $\leq V<3$. & 77.80 & 7.04 & 105.25 & 6.52 & 76.31 & 6.44 & 103.14 & 5.74 & 0.93 \\
\hline 3. $\leq V<4$. & 97.42 & 12.20 & 133.22 & 13.55 & 95.11 & 10.97 & 129.97 & 11.91 & 1.75 \\
\hline 4. $\leq V<5$. & 114.73 & 19.73 & 156.69 & 24.60 & 111.48 & 17.43 & 152.15 & 21.45 & 2.99 \\
\hline 5. $\leq V<6$. & 126.08 & 29.80 & 170.50 & 39.46 & 121.93 & 25.81 & 164.76 & 33.95 & 4.64 \\
\hline 6. $\leq V<7$. & 129.27 & 41.86 & 171.86 & 56.79 & 124.54 & 35.43 & 165.45 & 47.89 & 6.54 \\
\hline 7. $\leq V<8$. & 121.18 & 54.42 & 157.62 & 74.29 & 116.69 & 44.79 & 151.73 & 60.97 & 8.43 \\
\hline 8. $\leq V<9$. & 103.83 & 65.20 & 131.20 & 88.66 & 100.72 & 51.80 & 127.52 & 70.21 & 9.92 \\
\hline 9. $\leq V<10$. & 81.34 & 71.83 & 98.88 & 96.77 & 80.58 & 54.68 & 98.81 & 73.33 & 10.67 \\
\hline 10. $\leq V<11$ & 57.95 & 73.32 & 67.15 & 97.35 & 59.87 & 53.14 & 70.98 & 70.11 & 10.57 \\
\hline 11. $\leq V<12$ & 36.40 & 70.18 & 39.66 & 91.47 & 40.77 & 48.12 & 46.80 & 62.22 & 9.78 \\
\hline 12. $\leq V<13$. & 17.82 & 63.85 & 17.30 & 81.55 & 24.56 & 40.99 & 27.23 & 51.90 & 8.57 \\
\hline 13. $\leq V<14$. & 3.10 & 55.52 & 0.36 & 69.55 & 12.29 & 32.83 & 12.94 & 40.75 & 7.14 \\
\hline 14. $\leq V<15$ & -6.67 & 45.88 & -10.36 & 56.48 & 4.39 & 24.57 & 4.05 & 29.97 & 5.64 \\
\hline 15. $\leq V<16$ & -11.39 & 35.80 & -15.04 & 43.40 & 0.18 & 17.16 & -0.47 & 20.61 & 4.21 \\
\hline 16. $\leq V<17$ & -12.28 & 26.38 & -15.36 & 31.54 & -1.42 & 11.17 & -2.03 & 13.23 & 2.96 \\
\hline 17. $\leq V<18$ & -10.73 & 18.32 & -13.04 & 21.64 & -1.43 & 6.72 & -1.85 & 7.86 & 1.96 \\
\hline 18. $\leq V<19$ & -7.94 & 11.88 & -9.47 & 13.88 & -0.82 & 3.74 & -1.04 & 4.33 & 1.22 \\
\hline 19. $\leq V<20$ & -5.07 & 7.21 & -5.96 & 8.34 & -0.34 & 2.00 & -0.42 & 2.29 & 0.71 \\
\hline $20 . \leq V<21$ & -2.99 & 4.23 & -3.47 & 4.84 & -0.15 & 1.09 & -0.18 & 1.24 & 0.41 \\
\hline 21. $\leq V<22$ & -1.82 & 2.51 & -2.08 & 2.85 & -0.12 & 0.62 & -0.14 & 0.70 & 0.24 \\
\hline 22. $\leq V<23$ & -1.19 & 1.51 & -1.34 & 1.71 & -0.11 & 0.35 & -0.13 & 0.39 & 0.14 \\
\hline 23. $\leq V<24$ & -0.77 & 0.89 & -0.87 & 1.00 & -0.09 & 0.19 & -0.10 & 0.21 & 0.08 \\
\hline $24 . \leq V<25$ & -0.46 & 0.49 & -0.52 & 0.55 & -0.05 & 0.09 & -0.06 & 0.10 & 0.04 \\
\hline $25 . \leq V<26$ & -0.25 & 0.24 & -0.28 & 0.27 & -0.03 & 0.04 & -0.03 & 0.05 & 0.02 \\
\hline 26. $\leq V<27$ & -0.12 & 0.11 & -0.13 & 0.12 & -0.01 & 0.02 & -0.01 & 0.02 & 0.01 \\
\hline 27. $\leq V<28$ & -0.05 & 0.04 & -0.05 & 0.05 & 0.00 & 0.01 & -0.01 & 0.01 & 0.00 \\
\hline 28. $\leq V<29$ & -0.02 & 0.02 & -0.02 & 0.02 & 0.00 & 0.00 & 0.00 & 0.00 & 0.00 \\
\hline 29. $\leq V<30$. & -0.01 & 0.01 & -0.01 & 0.01 & 0.00 & 0.00 & 0.00 & 0.00 & 0.00 \\
\hline \multicolumn{10}{|c|}{ Direct sum } \\
\hline$\overline{V<0}$ & 79.28 & 1.71 & 95.05 & -0.48 & $\overline{78.83}$ & 1.63 & 94.46 & -0.57 & \\
\hline $0 \leq V<7$ & 652.30 & 116.36 & 875.80 & 144.44 & 635.00 & 101.41 & 851.85 & 123.96 & \\
\hline $7 \leq V<11$ & 364.30 & 264.78 & 454.84 & 357.07 & 357.86 & 204.42 & 449.04 & 274.62 & \\
\hline $11 \leq V<20$ & 3.24 & 335.03 & -11.90 & 417.84 & 78.18 & 187.29 & 85.21 & 233.17 & \\
\hline $20 \leq V<30$ & -7.67 & 10.04 & -8.77 & 11.42 & -0.57 & 2.41 & -0.66 & 2.72 & \\
\hline Total & 1091.45 & 727.92 & 1405.03 & 930.29 & 1149.30 & 497.15 & 1479.90 & 633.90 & \\
\hline \multicolumn{10}{|c|}{ Total value: $5126\left(|b|=90^{\circ}\right), 2789$ (slope) } \\
\hline
\end{tabular}


A.-L. Melchior et al.: The surface brightness of the Galaxy at the solar neighbourhood, Online Material p 4

Table A.2. Contributions from TYCHO-2 and USNO-A2 stars to the $B$ surface brightness, given in $10^{-9} \mathrm{~W} \mathrm{~m}^{-2}$. We give the values fitted to TYCHO-2 and USNO-A2 B data (see Figs. 10 and 11) for each sky area and magnitude bin. We also provide the corresponding values obtained with simulations.

\begin{tabular}{|c|c|c|c|c|c|c|c|c|}
\hline \multirow{3}{*}{$\begin{array}{r}B \text { mag. } \\
\text { Test }\end{array}$} & \multicolumn{4}{|c|}{ "Galactic centre } & \multicolumn{4}{|c|}{ Galactic anti-centre } \\
\hline & \multicolumn{2}{|c|}{ North } & \multicolumn{2}{|c|}{ South } & \multicolumn{2}{|c|}{ North } & \multicolumn{2}{|c|}{ South } \\
\hline & $|b|=90^{\circ}$ & slope & $|b|=90^{\circ}$ & slope & $|b|=90^{\circ}$ & slope & $|b|=90^{\circ}$ & slope \\
\hline \multicolumn{9}{|c|}{ TYCHO-2 data } \\
\hline $6 \leq B<7$ & 117.26 & 42.60 & 127.84 & 44.63 & 156.98 & 18.52 & 148.84 & 52.60 \\
\hline $7 \leq B<8$ & 144.37 & 36.62 & 143.04 & 45.85 & 154.85 & 34.38 & 157.57 & 53.65 \\
\hline $8 \leq B<9$ & 134.97 & 46.45 & 150.55 & 55.16 & 166.30 & 41.65 & 156.32 & 54.84 \\
\hline $9 \leq B<10$ & 147.35 & 47.23 & 151.01 & 64.20 & 164.94 & 50.75 & 158.00 & 56.83 \\
\hline $10 \leq B<11$ & 143.05 & 53.38 & 154.52 & 69.20 & 156.43 & 56.14 & 151.58 & 53.71 \\
\hline \multicolumn{9}{|c|}{ USNO-A2 data } \\
\hline $12 \leq B<13$ & 98.71 & 71.33 & 172.82 & 60.27 & 105.69 & 56.87 & 129.03 & 46.31 \\
\hline $13 \leq B<14$ & 66.24 & 64.01 & 88.08 & 80.64 & 85.80 & 49.79 & 93.22 & 41.05 \\
\hline $14 \leq B<15$ & 44.11 & 61.04 & 36.34 & 95.43 & 60.89 & 45.45 & 60.29 & 40.86 \\
\hline $15 \leq B<16$ & 24.81 & 59.49 & 2.90 & 102.94 & 43.99 & 40.07 & 34.36 & 39.84 \\
\hline $16 \leq B<17$ & 10.97 & 54.41 & -12.62 & 97.11 & 23.10 & 33.13 & 16.33 & 33.32 \\
\hline $17 \leq B<18$ & -2.58 & 49.07 & -15.81 & 79.41 & 7.92 & 24.74 & 6.19 & 24.73 \\
\hline $18 \leq B<19$ & -5.50 & 36.95 & -11.52 & 58.80 & 3.29 & 13.60 & 0.43 & 15.43 \\
\hline \multicolumn{9}{|c|}{ Simulations } \\
\hline $6 . \leq B<7$ & 134.14 & 36.53 & 177.34 & 49.10 & 129.41 & 30.81 & 170.95 & 41.20 \\
\hline 7. $\leq B<8$. & 132.53 & 46.41 & 171.57 & 62.97 & 127.44 & 38.26 & 164.90 & 51.72 \\
\hline 8. $\leq B<9$. & 122.53 & 55.15 & 154.82 & 74.66 & 117.63 & 44.19 & 148.74 & 59.60 \\
\hline 9. $\leq B<10$ & 105.65 & 61.44 & 129.94 & 82.38 & 101.49 & 47.55 & 125.28 & 63.46 \\
\hline 10. $\leq B<11$ & 83.91 & 64.51 & 100.27 & 85.24 & 80.90 & 47.96 & 97.53 & 62.99 \\
\hline 11. $\leq B<12$ & 59.73 & 64.27 & 69.13 & 83.45 & 58.41 & 45.54 & 68.77 & 58.72 \\
\hline 12. $\leq B<13$ & 35.89 & 61.02 & 39.71 & 77.81 & 37.20 & 40.65 & 42.65 & 51.44 \\
\hline $13 . \leq B<14$ & 15.42 & 55.09 & 15.26 & 69.05 & 20.32 & 33.80 & 22.46 & 42.04 \\
\hline 14. $\leq B<15$ & 0.95 & 46.83 & -1.46 & 57.79 & 9.22 & 25.94 & 9.60 & 31.74 \\
\hline 15. $\leq B<16$ & -6.86 & 37.20 & -9.94 & 45.23 & 3.09 & 18.39 & 2.81 & 22.17 \\
\hline 16. $\leq B<17$. & -9.61 & 27.73 & -12.42 & 33.25 & 0.16 & 12.20 & -0.27 & 14.49 \\
\hline 17. $\leq B<18$ & -9.52 & 19.62 & -11.73 & 23.22 & -0.95 & 7.62 & -1.33 & 8.94 \\
\hline 18. $\leq B<19$. & -8.03 & 13.22 & -9.62 & 15.47 & -1.05 & 4.46 & -1.31 & 5.17 \\
\hline \multicolumn{9}{|c|}{ Data Sums } \\
\hline $6 \leq B<11$ & 687.00 & 226.28 & 772.31 & 271.63 & 799.50 & 201.44 & 726.96 & 279.04 \\
\hline $12 \leq B<19$ & 236.76 & 396.30 & 260.19 & 574.60 & 330.68 & 263.65 & 339.85 & 241.54 \\
\hline \multicolumn{9}{|c|}{ Simulated Sums } \\
\hline $6 \leq B<11$ & 578.76 & 264.03 & 733.94 & 354.35 & 556.88 & 208.77 & 707.41 & 278.97 \\
\hline $12 \leq B<19$ & 18.24 & 260.71 & 9.79 & 321.82 & 67.99 & 143.07 & 74.60 & 175.99 \\
\hline \multicolumn{9}{|c|}{ Total contributions } \\
\hline & \multicolumn{4}{|c|}{ Data } & \multicolumn{4}{|c|}{ Simulations } \\
\hline & \multicolumn{2}{|c|}{$|b|=90^{\circ}$} & \multicolumn{2}{|c|}{ slope } & \multicolumn{2}{|c|}{$|b|=90^{\circ}$} & \multicolumn{2}{|c|}{ slope } \\
\hline$-6 \leq B<6$ & \multirow{2}{*}{\multicolumn{2}{|c|}{2985.77}} & \multirow{2}{*}{\multicolumn{2}{|c|}{978.39}} & 2841 & & 295 & \\
\hline $6 \leq B<11$ & & & & & 2577 & & 1106 & \\
\hline $11 \leq B<12$ & (98. & & (225. & & 256. & & 251 & \\
\hline $12 \leq B<19$ & $116^{7}$ & & 1476 & & 170. & & 901 & \\
\hline $6 \leq B<19$ & 410 & & 268 & & 5845 & & 2555 & \\
\hline $6 \leq B<11$ and $12 \leq B<19$ & 415 & & 245 & & 5845 & & 2555 & \\
\hline $6 \leq B<19$ & $(725$ & & $(300$ & & 5845 & 42. & 2555 & \\
\hline
\end{tabular}


A.-L. Melchior et al.: The surface brightness of the Galaxy at the solar neighbourhood, Online Material p 5

Table A.3. USNO-A2 R fluxes, given in $10^{-9} \mathrm{~W} \mathrm{~m}^{-2}$. We give the values fitted to USNO-A2 R fluxes (see Fig. 12 ) for $1 / \sin (b)<6$.

\begin{tabular}{|c|c|c|c|c|c|c|c|c|}
\hline \multirow{3}{*}{$R$ mag. } & \multicolumn{4}{|c|}{ Galactic centre } & \multicolumn{4}{|c|}{ Galactic anti-centre } \\
\hline & \multicolumn{2}{|c|}{ North } & \multicolumn{2}{|c|}{ South } & \multicolumn{2}{|c|}{ North } & \multicolumn{2}{|c|}{ South } \\
\hline & $|b|=90^{\circ}$ & slope & $|b|=90^{\circ}$ & slope & $|b|=90^{\circ}$ & slope & $|b|=90^{\circ}$ & slope \\
\hline \multicolumn{9}{|c|}{ USNO-A2 data } \\
\hline $12 \leq R<13$ & 79.69 & 145.06 & 100.21 & 202.54 & 92.32 & 95.03 & 94.15 & 84.65 \\
\hline $13 \leq R<14$ & 37.06 & 150.61 & 4.46 & 244.76 & 64.51 & 89.38 & 86.42 & 74.08 \\
\hline $14 \leq R<15$ & 13.02 & 129.08 & -38.66 & 212.66 & 48.15 & 71.91 & 41.65 & 68.61 \\
\hline $15 \leq R<16$ & 1.80 & 110.32 & -53.84 & 182.94 & 26.44 & 61.73 & 22.37 & 56.58 \\
\hline $16 \leq R<17$ & -8.92 & 88.87 & -48.69 & 143.69 & 8.12 & 43.45 & 7.76 & 40.04 \\
\hline $17 \leq R<18$ & -14.86 & 77.51 & -47.94 & 121.75 & -1.23 & 30.52 & 1.66 & 29.04 \\
\hline \multicolumn{9}{|c|}{ Simulations } \\
\hline$-6 . \leq R<-5$. & 2.24 & 0.02 & 2.47 & -0.02 & 2.24 & 0.02 & 2.47 & $-\mathbf{0 . 0 3}$ \\
\hline$-5 . \leq R<-4$. & 4.19 & 0.04 & 4.62 & -0.04 & 4.18 & 0.03 & 4.61 & -0.05 \\
\hline$-4 . \leq R<-3$. & 8.03 & 0.08 & 9.06 & -0.11 & 8.01 & 0.08 & 9.04 & -0.11 \\
\hline$-3 . \leq R<-2$. & 16.20 & 0.22 & 18.77 & -0.25 & 16.15 & 0.21 & 18.70 & -0.25 \\
\hline$-2 . \leq R<-1$. & 25.31 & 0.43 & 30.31 & -0.39 & 25.19 & 0.42 & 30.15 & -0.40 \\
\hline$-1 . \leq R<0$. & 37.62 & 1.02 & 46.91 & -0.24 & 37.36 & 0.98 & 46.55 & -0.28 \\
\hline $0 . \leq R<1$. & 51.95 & 2.17 & 67.20 & 0.69 & 51.40 & 2.05 & 66.43 & 0.56 \\
\hline 1. $\leq R<2$. & 68.12 & 4.62 & 91.55 & 3.37 & 67.05 & 4.32 & 90.05 & 3.00 \\
\hline 2. $\leq R<3$. & 88.53 & 8.91 & 121.29 & 8.90 & 86.63 & 8.22 & 118.61 & 8.00 \\
\hline 3. $\leq R<4$. & 107.01 & 16.05 & 147.62 & 19.07 & 104.05 & 14.53 & 143.44 & 17.01 \\
\hline 4. $\leq R<5$. & 120.52 & 26.40 & 165.66 & 34.26 & 116.51 & 23.33 & 160.06 & 30.03 \\
\hline 5. $\leq R<6$. & 124.63 & 39.66 & 169.40 & 53.60 & 120.13 & 34.08 & 163.17 & 45.86 \\
\hline 6. $\leq R<7$. & 117.68 & 54.20 & 157.03 & 74.33 & 113.76 & 45.12 & 151.73 & 61.71 \\
\hline 7. $\leq R<8$. & 99.92 & 67.51 & 129.94 & 92.73 & 97.98 & 54.22 & 127.56 & 74.30 \\
\hline 8. $\leq R<9$. & 76.71 & 76.42 & 95.67 & 104.57 & 77.90 & 58.91 & 97.90 & 80.35 \\
\hline 9. $\leq R<10$. & 53.41 & 79.19 & 62.59 & 107.30 & 58.05 & 58.28 & 69.87 & 78.60 \\
\hline 10. $\leq R<11$ & 33.95 & 75.96 & 36.46 & 101.35 & 41.17 & 53.22 & 47.42 & 70.51 \\
\hline 11. $\leq R<12$ & 18.59 & 68.85 & 17.45 & 90.00 & 27.17 & 45.78 & 30.11 & 59.31 \\
\hline 12. $\leq R<13$ & 6.01 & 60.16 & 3.06 & 76.90 & 15.59 & 37.60 & 16.55 & 47.60 \\
\hline 13. $\leq R<14$ & -3.99 & $\mathbf{5 0 . 9 6}$ & -7.85 & 63.78 & 6.85 & 29.40 & 6.65 & 36.45 \\
\hline 14. $\leq R<15$. & -10.34 & 41.37 & -14.40 & $\mathbf{5 0 . 8 5}$ & 1.42 & 21.62 & 0.72 & 26.33 \\
\hline 15. $\leq R<16$ & -12.74 & 31.82 & -16.40 & 38.51 & -1.06 & 14.82 & -1.81 & 17.77 \\
\hline 16. $\leq R<17$. & -12.02 & 23.04 & -14.90 & 27.50 & -1.50 & 9.41 & -2.06 & 11.13 \\
\hline 17. $\leq R<18$ & -9.47 & 15.63 & -11.47 & 18.43 & -0.99 & 5.55 & -1.30 & 6.48 \\
\hline 18. $\leq R<19$ & -6.46 & 9.99 & -7.69 & 11.64 & -0.42 & 3.13 & -0.55 & 3.61 \\
\hline 19. $\leq R<20$ & -4.04 & 6.16 & -4.73 & 7.11 & -0.18 & 1.78 & -0.22 & 2.03 \\
\hline 20. $\leq R<21$ & -2.58 & 3.83 & -2.98 & 4.37 & -0.17 & 1.05 & -0.20 & 1.19 \\
\hline 21. $\leq R<22$ & -1.81 & 2.45 & -2.06 & 2.77 & -0.19 & 0.62 & -0.22 & 0.71 \\
\hline \multicolumn{9}{|c|}{ Data sums } \\
\hline $12 \leq R<18$ & 107.79 & 701.45 & -84.46 & 1108.34 & 238.31 & 392.02 & 254.01 & 353.00 \\
\hline \multicolumn{9}{|c|}{ Simulated sums } \\
\hline$-6 \leq R<6$ & 654.35 & 99.62 & 874.86 & 118.84 & 638.89 & 88.26 & 853.29 & 103.34 \\
\hline $6 \leq R<12$ & 400.26 & 422.13 & 499.16 & 570.30 & 416.02 & 315.53 & 524.58 & 424.79 \\
\hline $12 \leq R<18$ & -42.56 & 222.99 & -61.97 & 275.96 & 20.31 & 118.39 & 18.74 & 145.75 \\
\hline $18 \leq R<22$ & -14.88 & 22.43 & -17.46 & 25.90 & -0.96 & 6.58 & -1.19 & 7.54 \\
\hline \multicolumn{9}{|c|}{ Total contributions } \\
\hline & \multicolumn{4}{|c|}{ Data } & \multicolumn{4}{|c|}{ Simulations } \\
\hline & \multicolumn{2}{|c|}{$|b|=90^{\circ}$} & \multicolumn{2}{|c|}{ slope } & \multicolumn{2}{|c|}{$|b|=90^{\circ}$} & \multicolumn{2}{|c|}{ slope } \\
\hline$-6 \leq R<6$ & & & & & 302 & & 410 & \\
\hline $6 \leq R<12$ & & & & & 184 & & 173 & \\
\hline $12 \leq R<18$ & 515 & & 25 & & -65 & & 763 & \\
\hline $18 \leq R<22$ & & & & & -34 & & 62 & \\
\hline $6 \leq R<22$ & & & & & 476 & & 296 & \\
\hline
\end{tabular}


Table A.4. Values of the surface brightness computed with the COBE/DIRBE data, given in $10^{-9} \mathrm{~W} \mathrm{~m}^{-2}$. We give the values fitted to the COBE/DIRBE data for $1 / \sin |b|<6$. The influence of point sources is removed with a $5 \sigma$ clipping. The slopes are computed as explained for the optical (for $1 / \sin |b|<6$ ), except for the 12 and $25 \mu \mathrm{m}$ data. As explained in the text (Sect. 5), these data exhibit a "non-Galactic behaviour" and the corresponding fits are performed for $2<1 / \sin |b|<6$.

\begin{tabular}{|c|c|c|c|c|c|c|c|c|}
\hline \multirow{3}{*}{$\lambda$} & \multicolumn{4}{|c|}{$\overline{\text { Galactic centre }}$} & \multicolumn{4}{|c|}{ Galactic anti-centre } \\
\hline & \multicolumn{2}{|c|}{ North } & \multicolumn{2}{|c|}{ South } & \multicolumn{2}{|c|}{ North } & \multicolumn{2}{|c|}{ South } \\
\hline & $|b|=90^{\circ}$ & slope & $|b|=90^{\circ}$ & slope & $|b|=90^{\circ}$ & slope & $|b|=90^{\circ}$ & slope \\
\hline $1.25 \mu \mathrm{m}$ & 2050. & 2240. & 1720. & 2790. & 2180. & 1130. & 2320 . & 1330. \\
\hline $2.20 \mu \mathrm{m}$ & 964. & 1130. & 787. & 1390. & 1020. & 541. & 1050. & 676. \\
\hline $3.50 \mu \mathrm{m}$ & 397. & 393. & 333. & 472. & 408. & 191. & 417. & 252. \\
\hline $4.90 \mu \mathrm{m}$ & 324. & 155. & 305. & 171. & 317. & 77.8 & 345. & 97.3 \\
\hline $12 \mu \mathrm{m}$ & 1630. & 121. & 1500. & 86.7 & 979. & 181. & 1440. & 139. \\
\hline $25 \mu \mathrm{m}$ & 1260. & 57.5 & 1260. & 28.5 & 1020. & 69.1 & 1160. & 59.0 \\
\hline $60 \mu \mathrm{m}$ & 288. & 239. & 136. & 203. & 148. & 133. & 272. & 201. \\
\hline $100 \mu \mathrm{m}$ & 665. & 549. & 406. & 439. & 235. & 385. & 563. & 511. \\
\hline $140 \mu \mathrm{m}$ & 764. & 800. & 553. & 567. & 185. & 638. & 660. & 802. \\
\hline $240 \mu \mathrm{m}$ & 315. & 351. & 267. & 238. & 45.8 & 332. & 302. & 382. \\
\hline \multicolumn{9}{|c|}{ Contribution from the Galactic Centre (left) and Anti-Centre (right) } \\
\hline $1.25 \mu \mathrm{m}$ & \multicolumn{4}{|c|}{ 3782. $\left(|b|=90^{\circ}\right) 5040$. (slope) } & \multicolumn{4}{|c|}{ 4500. $\left(|b|=90^{\circ}\right) 2462$. (slope) } \\
\hline $2.20 \mu \mathrm{m}$ & \multirow{2}{*}{\multicolumn{4}{|c|}{$\begin{aligned} 1752 .(|b| & \left.=90^{\circ}\right) 2520 . \text { (slope) } \\
730 .(|b| & \left.=90^{\circ}\right) 864 . \text { (slope) }\end{aligned}$}} & \multicolumn{4}{|c|}{ 2066. $\left(|b|=90^{\circ}\right) 1216$. (slope) } \\
\hline $3.50 \mu \mathrm{m}$ & & & & & \multicolumn{4}{|c|}{ 826. $\left(|b|=90^{\circ}\right)$ 442. (slope) } \\
\hline $4.90 \mu \mathrm{m}$ & \multicolumn{4}{|c|}{$\begin{array}{l}\text { 730. }\left(|b|=90^{\circ}\right) 864 . \text { (slope) } \\
630 .\left(|b|=90^{\circ}\right) 324 . \text { (slope) }\end{array}$} & \multicolumn{4}{|c|}{ 662. $\left(|b|=90^{\circ}\right)$ 174. (slope) } \\
\hline $12 \mu \mathrm{m}$ & \multicolumn{4}{|c|}{$\begin{array}{l}\text { 630. }\left(|b|=90^{\circ}\right) 324 \text {. (slope) } \\
\text { 3126. }\left(|b|=90^{\circ}\right) 208 . \text { (slope) }\end{array}$} & \multicolumn{4}{|c|}{ 2418. $\left(|b|=90^{\circ}\right) 320$. (slope) } \\
\hline $25 \mu \mathrm{m}$ & \multicolumn{4}{|c|}{ 2522. $\left(|b|=90^{\circ}\right) 86$. (slope) } & \multicolumn{4}{|c|}{ 2182. $\left(|b|=90^{\circ}\right) 128$. (slope) } \\
\hline $60 \mu \mathrm{m}$ & \multicolumn{4}{|c|}{ 424. $\left(|b|=90^{\circ}\right)$ 442. (slope) } & \multicolumn{4}{|c|}{ 420. $\left(|b|=90^{\circ}\right)$ 332. (slope) } \\
\hline $100 \mu \mathrm{m}$ & \multicolumn{4}{|c|}{ 1070. $\left(|b|=90^{\circ}\right) 988$. (slope) } & \multicolumn{4}{|c|}{ 800. $\left(|b|=90^{\circ}\right) 894$. (slope) } \\
\hline $140 \mu \mathrm{m}$ & \multicolumn{4}{|c|}{ 1318. $\left(|b|=90^{\circ}\right) 1366$. (slope) } & \multicolumn{4}{|c|}{ 844. $\left(|b|=90^{\circ}\right) 1440$.(slope) } \\
\hline $240 \mu \mathrm{m}$ & \multicolumn{4}{|c|}{ 584. $\left(|b|=90^{\circ}\right) 590$. (slope) } & \multicolumn{4}{|c|}{ 246. $\left(|b|=90^{\circ}\right) 714$. (slope) } \\
\hline & & Surface & rightness c & mputed & ver the wh & le sky & & \\
\hline $1.25 \mu \mathrm{m}$ & & & 8282. & $\overline{b \mid}=90$ & $7502 .(\mathrm{slc}$ & & & \\
\hline $2.20 \mu \mathrm{m}$ & & & 3818 & $|b|=90$ & 3736. (slc & & & \\
\hline $3.50 \mu \mathrm{m}$ & & & 1556 & $|b|=90$ & 1306. (slc & & & \\
\hline $4.90 \mu \mathrm{m}$ & & & 1292 & $|b|=9$ & 500. ( $\mathrm{slo}$ & & & \\
\hline $12 \mu \mathrm{m}$ & & & 5544 & $|b|=9$ & 528. (slo & & & \\
\hline $25 \mu \mathrm{m}$ & & & 4704 & $|b|=9$ & ) 214. ( slo & & & \\
\hline $60 \mu \mathrm{m}$ & & & 844 & $|b|=90$ & ) 774. (slor & & & \\
\hline $100 \mu \mathrm{m}$ & & & 1870 & $|b|=90$ & 1882. $(\mathrm{slc}$ & & & \\
\hline $140 \mu \mathrm{m}$ & & & 2162. & $|b|=90$ & 2806. ( slc & & & \\
\hline $240 \mu \mathrm{m}$ & & & 930. & $b \mid=90$ & 1304. (slo & & & \\
\hline
\end{tabular}




\section{Appendix B: Surface brightness definition}

Following the usual conventions, the surface brightness $v S_{v}$ is defined as:

$v S_{v}=\frac{L_{\lambda}}{\pi R^{2}}$

where $R$ is the radius (in $\mathrm{m}$ ) of the area probed and $L_{\lambda}$ its luminosity in $\mathrm{W}$ at the wavelength $\lambda . v S_{v}$ can be directly transformed to $L_{\lambda \odot} \mathrm{pc}^{-2}$. It can also be transformed to a magnitude scale $\mu$ (mag $\operatorname{arcsec}^{-2}$ ) with:

$\mu_{\lambda}=-2.5 \log _{10}\left[v S_{v} \times \frac{1}{c_{\lambda}} \times \frac{x_{\mathrm{sr} \rightarrow \operatorname{arcsec}^{2}}}{4 \pi}\right]$

where $x_{\mathrm{sr} \rightarrow \operatorname{arcsec}^{2}}=23.5 \times 10^{-12} \operatorname{arcsec}^{2} / \mathrm{sr}$ is the solid angle normalisation and $c_{\lambda}$ is the conversion factor of the observed surface brightness into magnitude. This conversion factor is for instance provided by $\mathrm{NED}^{5}$ For $B, V$ and $R$ bands, we thus have the following conversions:

$\mu_{B}=-2.5 \log _{10}\left[v_{B} S_{B} \times \frac{1}{2.90 \times 10^{-8}} \times \frac{23.5 \times 10^{-12}}{4 \pi}\right]$

$\mu_{V}=-2.5 \log _{10}\left[v_{V} S_{V} \times \frac{1}{1.97 \times 10^{-8}} \times \frac{23.5 \times 10^{-12}}{4 \pi}\right]$

$\mu_{R}=-2.5 \log _{10}\left[v_{R} S_{R} \times \frac{1}{1.44 \times 10^{-8}} \times \frac{23.5 \times 10^{-12}}{4 \pi}\right]$

with $v_{B} S_{B}, v_{V} S_{V}$ and $v_{R} S_{R}$ the surface brightness in $B, V$ and $R$ in $\mathrm{W} \mathrm{m}^{-2}$, while $\mu_{B}, \mu_{V}$ and $\mu_{R}$ are in $B$ mag arcsec ${ }^{-2}$, $V$ mag $\operatorname{arcsec}^{-2}$ and $R$ mag $\operatorname{arcsec}^{-2}$.

\section{Appendix C: Normalisation factor of the cosecant integration method}

We check in the simple case of a uniform distribution $\left(n\left(r, b, l, M_{V}\right)=1, f\left(A_{V}(r, b)\right)=1\right.$ and $\left.\phi\left(M_{V}\right) L\left(M_{V}\right)=1\right)$ the normalisation of the cosecant integration method with respect to the integration in a cylinder.

$S_{\mathrm{cyl}}^{\prime}=\left[\int_{R=0}^{R_{\mathrm{cyl}}} \int_{z=-z_{\max }}^{+z_{\max }} \int_{l=0}^{2 \pi} \mathrm{d} l \mathrm{~d} z R \mathrm{~d} R\right] / \pi R_{\mathrm{cyl}}^{2}=2 z_{\max }$

$S_{\text {cosec }}^{\prime}=\int_{b=-\frac{\pi}{2}}^{\frac{\pi}{2}} \int_{l=0}^{2 \pi} \int_{r=0}^{r_{\max }(b)} \frac{r^{2} \mathrm{~d} r \mathrm{~d} l \cos b \sin |b| d b}{4 \pi r^{2}}$

$$
=z_{\max }
$$

$S_{\text {cyl }}^{\prime}$ is the simplified version of the surface brightness computed as the integration in a cylinder, while $S_{\text {cosec }}^{\prime}$ corresponds to the integration with the $\sin |b|$ weighting used for the cosecant method. They differ by a factor of 2 understood as the difference of the volumes probed. As the behaviour of the exponential disc geometry is close to the plane parallel geometry (see e.g. Fig. 4), we apply this factor 2 to the normalisation of all estimates of the surface brightness based on the cosecant approximation, namely the integration in a cone (Eq. (3)), the corrected direct estimate (Eqs. (6) and (7)) and the cosecant compilations of the various datasets. We check that this factor does not change more than $7 \%$ with an exponential geometry.

${ }^{5}$ http://nedwww.ipac. caltech.edu/help/photoband.1st 


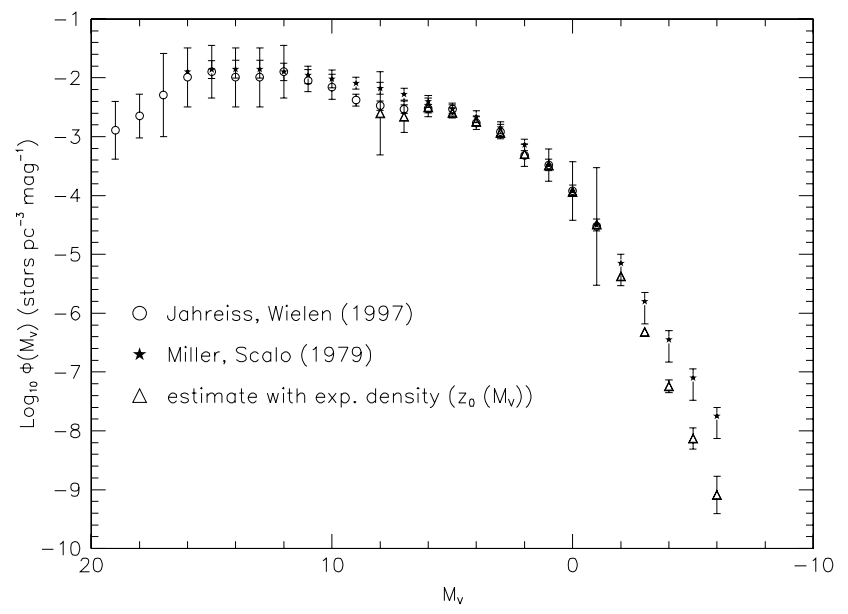

Fig. D.1. Luminosity function at the Solar Neighbourhood. The LF used for the simulation is presented (Miller \& Scalo 1979; Jahreiss \& Wielen 1997). The LF used for the direct/indirect estimation is also displayed with error bars accounting for Poisson noise only.

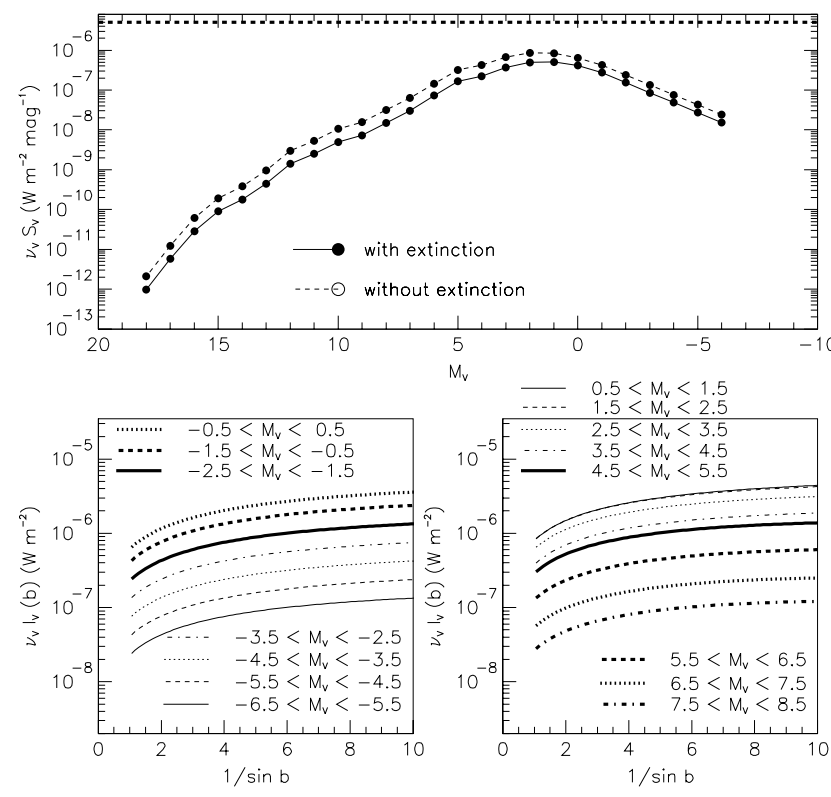

Fig. D.2. Contribution of each absolute magnitude bin to the $V$ surface brightness (computed with $1 / \sin |b|<6$ from the Solar Neighbourhood, with $h_{Z}\left(M_{V}\right)$ ). The upper panel displays the contribution to $v_{V} S_{V}$ of each bin of absolute magnitude. The horizontal dashed line corresponds to the cumulated value (the estimated surface brightness). The lower panels exhibit the corresponding decomposition of $v_{V} I_{V}(|b|)$ for each absolute-magnitude bin (with extinction). These values have to be increased by $65 \%$ (see Fig. 2) to correspond to a face-on value estimate of the $V$ surface brightness.

\section{Appendix D: Luminosity function (LF)}

We use two estimates of the LF to define $\phi\left(M_{V}\right)$ : (1) the Miller \& Scalo (1979) estimate for $M_{V}<2$, (2) the Jahreiss \& Wielen (1997) estimate based on Hipparcos data for $M_{V} \geq 2$. They are displayed in Fig. D.1. We find that this choice of the LF combined with the variable scale height $h_{Z}\left(M_{V}\right)$ of Miller \& Scalo (1979) is equivalent to a fixed scale height $h_{Z}=125 \mathrm{pc}$ (used to compare the plane parallel and exponential disc geometries).

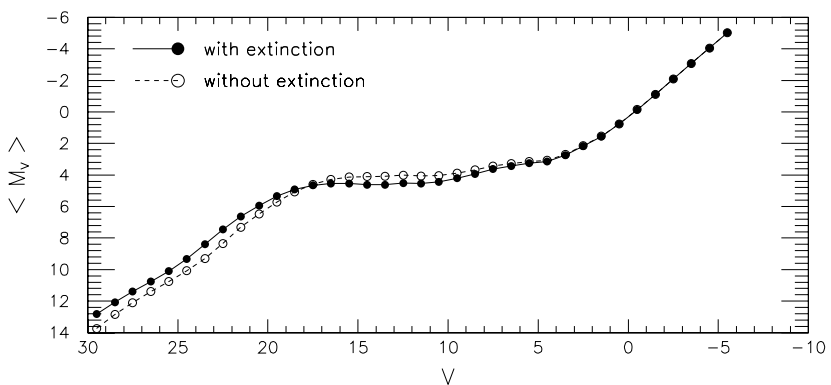

Fig. D.3. Mean absolute magnitude of stars contributing to each apparent magnitude bin.

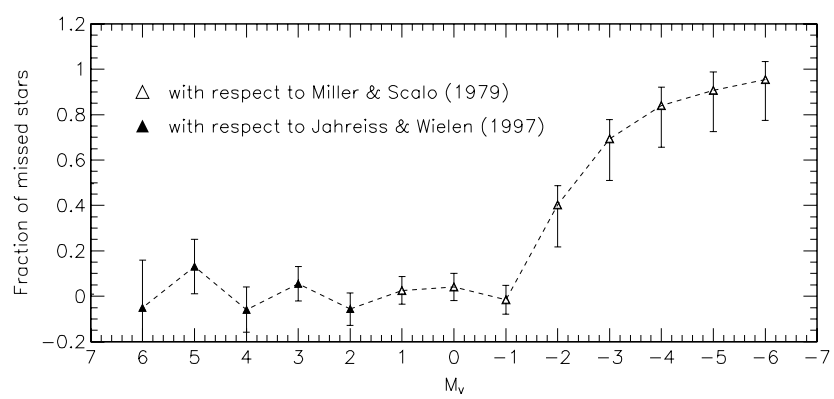

Fig. D.4. Fraction $f_{i}^{\text {missed }}$ of the stars missed with respect to Miller \& Scalo (1979) for each magnitude bin. The correction factor $f_{i}^{\mathrm{LF}}=$ $1 /\left(1-f_{i}^{\text {missed }}\right)$ is applied for the computation of the corrected and indirect estimates in Sect. 3 in order to be compatible with the simulations. The error bars account for the uncertainties in the Jahreiss \& Wielen (1997); Miller \& Scalo (1979) LF used in the simulations and for the Poisson noise of our estimate.

\section{D.1. Uncertainties}

Figure D. 2 shows the relative contribution to the surface brightness from the Solar Neighbourhood of each absolute-magnitude bin. This has been computed with the simulations described in Sect. 2.2. Dim stars with $M_{V}>7$ do not contribute significantly to the surface brightness $(1 \%)$, while the magnitude interval $-6 \leq M_{V} \leq 6$ is the main contributor to the surface brightness $(98 \%)$. It is important to note that the bright end $\left(M_{V}<0.5\right)$ of the LF constitutes a significant contribution (34\% of the surface brightness). Hence, theoretical estimations of the surface brightness based on the LF depend on the uncertainties on the upper tail. However, stars with $M_{V}<-1.5$ (resp. $M_{V}<-3.5$ ) correspond to only $11 \%$ (resp. $3 \%$ ) of the surface brightness. This effect disappears when one considers stellar catalogues, which provide apparent magnitudes (see Fig. 8). Figure D.2 displays the value of the absolute magnitude of the "mean star" of each apparent magnitude bin, which illustrates qualitatively the behaviour of these related parameters.

\section{D.2. Computation of the $L F$ for direct/indirect estimates}

We present here the estimate of the LF, based on the Hipparcos catalogue, used to compute the direct and indirect estimates. The Hipparcos Catalogue (Perryman et al. 1997; van Leeuwen et al. 1997) is complete for $V<7.3$, and parallaxes are available for most of the stars. We consider that the number of stars in each magnitude bin $M_{V}-\frac{1}{2} \leq m<M_{V}+\frac{1}{2}$, where $m$ is the absolute magnitude, is complete within a sphere of radius 
$R\left(M_{V}+\frac{1}{2}\right)=10^{\left(7.3-\left(M_{V}+\frac{1}{2}\right)\right) / 5 .+1} \mathrm{pc}$ for $-6.5 \leq M_{V}<6.5$. We count stars in these volumes $\operatorname{vol}(R)$ using,

$\phi\left(M_{V}\right)=\frac{N\left(M_{V}-\frac{1}{2} \leq m<M_{V}+\frac{1}{2}\right)}{\operatorname{vol}(R)}$

where $N\left(M_{V}-\frac{1}{2} \leq m<M_{V}+\frac{1}{2}\right)$ is the number of stars found with an absolute magnitude $m$ in this bin, and the volume is defined as: $\operatorname{vol}(R)=\int_{r<R} \eta\left(r, b, l ; h_{Z}\left(M_{V}\right)\right) r^{2} \mathrm{~d} \sin b \mathrm{~d} l \mathrm{~d} r$, and where $\eta\left(r, b, l ; h_{Z}\left(M_{V}\right)\right)=n\left(r, b, l, M_{V}\right) / n_{\odot}\left(M_{V}\right)$ (open triangles in Fig. D.1). This accounts for the exponential profile of the Galaxy, with different vertical scales for different magnitude ranges. We adopt the same scale heights as Miller \& Scalo (1979) $\left(h_{Z}\left(M_{V} \leq 0\right)=80 \mathrm{pc}\right)$. We also correct each star for interstellar extinction according to our extinction modelling described in Appendix E.

Among the 20906 stars with $V<7.3$, we consider the 18381 stars with a parallax significant at $3 \sigma$. We further restrict this sample by $29 \%$ to account the completion limit for each magnitude bin. We then treat the 13374 stars as follows:

- 9444 stars have a standard spectral type with a luminosity class that can be found in the Pickles (1998) library.

- 548 stars either have a standard spectral type with no luminosity class available in Pickles (1998) and that has to be interpolated between existing templates, or have two spectral types (the average is then adopted).

- 3114 stars have a well defined spectral type, but no luminosity class. We rely on $M_{V}$ to estimate it, and derive a template.

- 1 Wolf-Rayet star and $20 \mathrm{C} / \mathrm{N}$ stars, which do not exceed $3.06 \times 10^{-8} \mathrm{~W} \mathrm{~m}^{-2} \mathrm{sr}^{-1}$ (corrected direct) and $1.40 \times$ $10^{-9} \mathrm{~W} \mathrm{~m}^{-2} \mathrm{sr}^{-1}$ (indirect) in $V$.
- 247 stars, with no precise spectral type (63\% Am/Ap, $18 \% \mathrm{M})$, are neglected. We estimate that their contribution to the $V$ surface brightness is $6.80 \times 10^{-8} \mathrm{~W} \mathrm{~m}^{-2} \mathrm{sr}^{-1}$ (corrected direct) and $5.69 \times 10^{-9} \mathrm{~W} \mathrm{~m}^{-2} \mathrm{sr}^{-1}$ (indirect).

Figure D.1 summarises the LF thereby obtained. The discrepancy with respect to Miller \& Scalo (1979) is significant for luminous stars. The corresponding stellar population is strongly inhomogeneous (de Zeeuw et al. 1999), and this part of the LF certainly suffers uncertainties larger than Poisson noise (see also Appendix D.3). In order, to use the same LF as for the simulations, we correct the LF as displayed in Fig. D.4, for stars brighter than magnitude -1.5 .

\section{D.3. $3 D$ visualisation of the Hipparcos data}

We present a 3D visualisation of the Hipparcos catalogue on http://www.obspm.fr/sbhip06. We also position a schematic Gould's belt in the Galactic plane following Guillout et al. (1998). Gould's belt is significantly larger than the volume probed by the Hipparcos catalogue. The early-type stars are distributed in a disc slightly inclined with respect to the Galactic Disc $(15 \pm 7 \mathrm{deg})$. More generally, the Hipparcos stars' distribution is clearly flatten towards the Galactic Poles, but no clear correlation with Gould's belt is detected due to the small volume sampled. For the majority of the stars, the cut in magnitude is clearly seen as a spheroidal distribution of stars. However, the bright stars exhibit a more irregular distribution. The apparent contours are roughly compatible with the local cavity observed in the gas distribution (e.g. Lallement et al. 2003), but there is not enough information to draw conclusions about an extinction bias. 

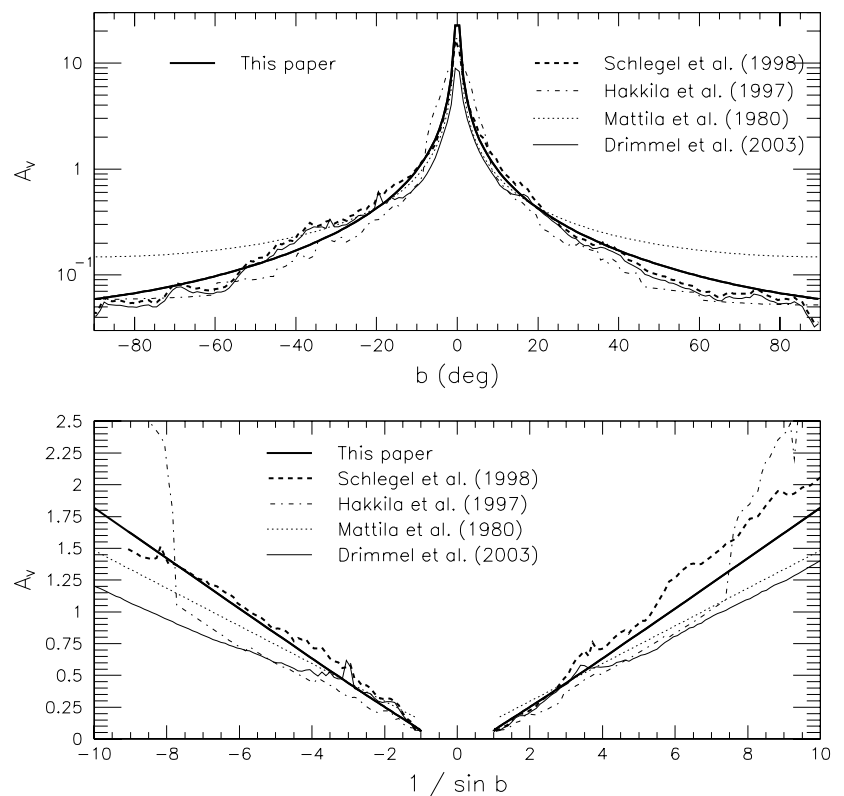

Fig. E.1. $A_{V}$ extinction dependence on Galactic latitude. The extinction used for the simulations is compared with other models (Drimmel et al. 2003; Hakkila et al. 1997; Mattila 1980b) and the dust maps (Schlegel et al. 1998).

Table E.1. Values of the extinction correction applied to cosecant measurements for the main filters used in this paper (see Fig. 2 and Table E.1). We consider $R_{\text {cyl }}=1 \mathrm{kpc}$.

\begin{tabular}{rr|rr}
\hline \hline$\lambda(\mu \mathrm{m})$ & Correction & $\lambda(\mu \mathrm{m})$ & Correction \\
\hline $0.44(B)$ & 1.72 & $0.69\left(R_{\mathrm{J}}\right)$ & 1.36 \\
$0.55(V)$ & 1.52 & $1.25(J)$ & 1.12 \\
$0.64\left(R_{\mathrm{C}}\right)$ & 1.41 & $2.20(K)$ & 1.05 \\
\hline
\end{tabular}

\section{Appendix E: Extinction modelling}

In Fig. E.1, we display the dependence of the $A_{V}$ extinction on Galactic latitude obtained from dust maps by Schlegel et al. (1998). We find systematic differences with Hakkila et al. (1997), based on a compilation of various models. For the purpose of this work, we wish to use a simple analytical model to describe first order effects linked to extinction. We consider the extinction model of Mattila (1980b), based on a 2-components model. Compared to the $A_{V}$ extinction derived from Schlegel et al. (1998), it tends to overestimate the extinction towards the pole (even though this excess remains within the error bars quoted by Hakkila et al. 1997), and to underestimate it at intermediate latitudes. We further tune this type of law, and adopt the following empirical model:

$A_{V}(r, b)=a_{v} \beta / \sin |b| \times\left(\frac{\pi}{b+\pi}\right)^{3} \times(1-\exp (-(r|\sin b|) / \beta))(\mathrm{E} .1)$

with $a_{v}=1.48 \mathrm{mag} / \mathrm{kpc}$ and $\beta=135 \mathrm{pc}$. The inverse cube power of $b$ has been defined empirically to reproduce the $b$ dependence in Fig. E.1. This empirical law is also in good agreement with the $z$-dependence of the extinction modelled by Hakkila et al. (1997).

\section{Numerical values}

The extinction correction used to derive a surface brightness measurement of the Galaxy at the Solar Neighbourhood is provided in Table E.1. 
A.-L. Melchior et al.: The surface brightness of the Galaxy at the solar neighbourhood, Online Material p 11

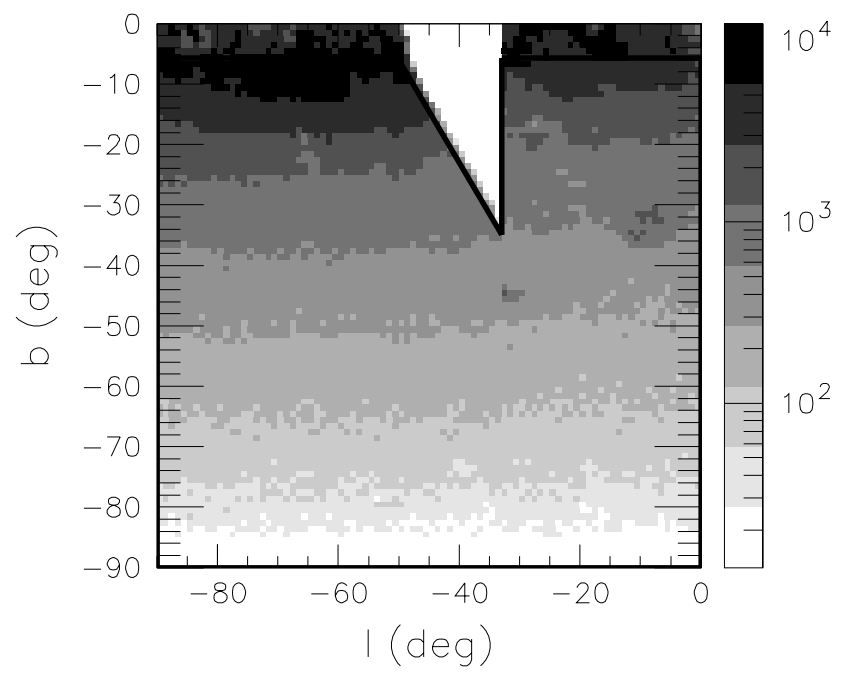

Fig. F.1. Galactic plane coverage of the UCAC-1 catalogue (Zacharias et al. 2000). We restrict our study to the area indicated with thick lines, defined by $b<0$ and $-90^{\circ}<l<0^{\circ}$, and we exclude the area between $l=-33^{\circ}$ and $b=-1.7 \times l+522^{\circ}$. The intensity corresponds to the number of stars with $11 \leq R_{U}<16$.

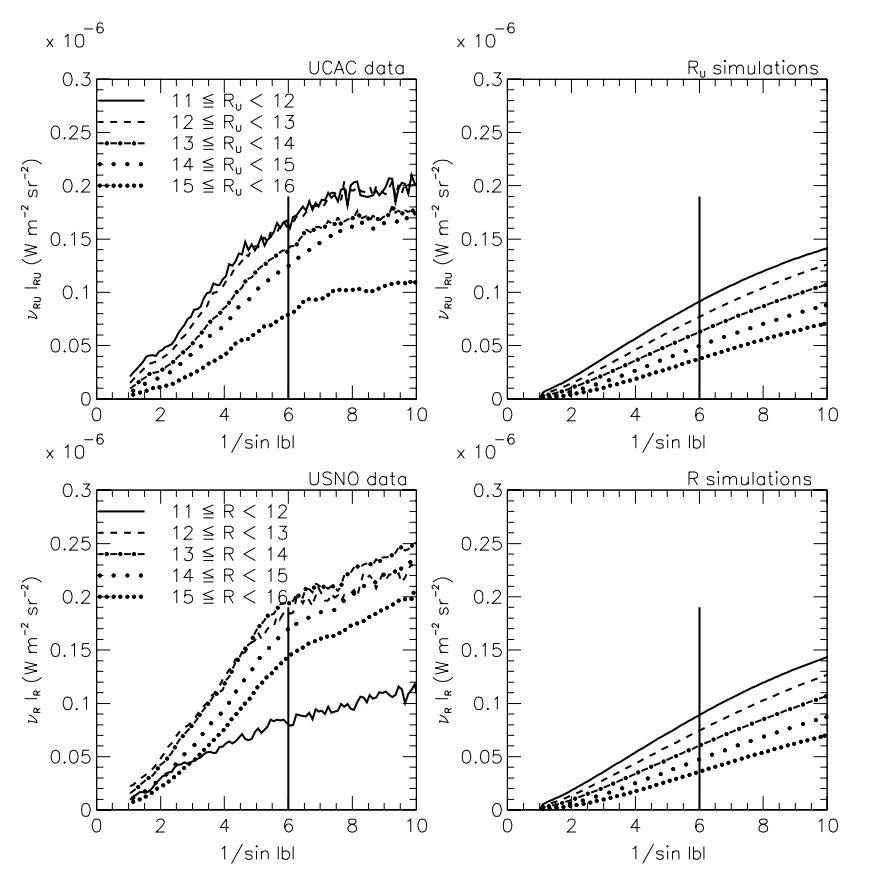

Fig. F.2. Integrated $R$ flux as a function of $1 / \sin (|b|)$ for the UCAC (top) and USNO-A2 (bottom) stars lying in the UCAC-1 area defined in Fig. F.1. These fluxes correspond to the contribution of this area to the total flux received at the Sun. No correction has been applied. The results of the fits for $1 / \sin (|b|)<6$ are provided in Table F.1.

\section{Appendix F: Comparison of the USNO-A2 with UCAC-1 data}

Figure F.1 displays the area of the sky considered for this comparison. Figure F. 2 and Table F.1 compare the 2 data sets with simulations.
Table F.1. $R_{U}$ (UCAC-1) and $R$ (USNO-A2) fluxes, given in $10^{-9} \mathrm{~W} \mathrm{~m}^{-2} \mathrm{sr}^{-1}$. We give the values (2) and (3) fitted to $R_{U}$ (UCAC-1) and $R$ (USNO-A2) fluxes for $1 / \sin |b|<6$ (see Fig. E.1) over the area defined in Fig. F.1, for each magnitude bin (1). Last, we provide the ratio (4) between the UCAC-1 and USNO-A2 slopes. No correction is applied.

\begin{tabular}{|c|c|c|c|c|}
\hline $\begin{array}{r}\text { Magnitude range } \\
\text { (1) }\end{array}$ & $\begin{array}{r}|b|=90^{\circ} \\
\text { (2) }\end{array}$ & $\begin{array}{r}\text { Slope } \\
\text { (3) }\end{array}$ & $\begin{array}{r}|b|=90^{\circ} \\
\text { (4) }\end{array}$ & $\begin{array}{r}\text { Slope } \\
\text { (5) }\end{array}$ \\
\hline & \multicolumn{2}{|c|}{ UCAC-1 data } & \multicolumn{2}{|c|}{$R_{U}$ Simulation } \\
\hline $8 \leq R_{U}<9$ & 22.49 & 8.07 & 7.32 & 31.20 \\
\hline $9 \leq R_{U}<10$ & 31.98 & 16.38 & 5.74 & 26.86 \\
\hline $10 \leq R_{U}<11$ & 26.88 & 25.62 & 4.22 & 22.22 \\
\hline $11 \leq R_{U}<12$ & 17.56 & 31.55 & 2.87 & 17.50 \\
\hline $12 \leq R_{U}<13$ & 7.72 & 32.98 & 1.80 & 12.99 \\
\hline $13 \leq R_{U}<14$ & 0.03 & 28.88 & 1.03 & 9.06 \\
\hline $14 \leq R_{U}<15$ & -3.95 & 25.61 & 0.55 & 5.90 \\
\hline \multirow[t]{2}{*}{$15 \leq R_{U}<16$} & -4.87 & 16.36 & 0.29 & 3.59 \\
\hline & \multicolumn{2}{|c|}{ USNO-A2 data } & \multicolumn{2}{|c|}{$R_{C}$ Simulation } \\
\hline $11 \leq R<12$ & 13.51 & 15.16 & 2.87 & 17.50 \\
\hline $12 \leq R<13$ & 14.94 & 35.80 & 1.80 & 12.99 \\
\hline $13 \leq R<14$ & 4.00 & 39.58 & 1.03 & 9.06 \\
\hline $14 \leq R<15$ & -4.45 & 34.63 & 0.55 & 5.90 \\
\hline $15 \leq R<16$ & -7.39 & 29.38 & 0.29 & 3.59 \\
\hline
\end{tabular}




\section{Appendix G: Calibration of the USNO-A2 data}

Given the dispersion of USNO-A2 magnitudes, accounting for colour effects would introduce an additional source of noise for single wavelength flux measurements. As we are interested in integrating the total flux at given wavelengths, we deliberately neglect colour effects and perform the calibration between single filters. The red measurements correspond to the plate emulsions 103a-E (North) and IIIa-F (south). The equivalent wavelengths are respectively $6450 \AA$ and $6400 \AA$ which are very close to the $R_{C}$ filter. The blue measurements (103a-O (POSS-I O) for the North, and IIIa-J (POSS-I E) for the south) differ more significantly with equivalent wavelengths of $4050 \AA$ and $4680 \AA$. We estimate that a $10 \%$ uncertainty is introduced when aligning these measurements to the $B_{J}$ data, which remains negligible for our purposes.

We cross-identify the 526280881 USNO-A2 stars (Monet et al. 1998) with those of the GSPC2.1 catalogue (Bucciarelli et al. 2001) composed of 305017 stars with CCD (B)VR photometry within a $5^{\prime \prime} \times 5^{\prime \prime}$ window. In the USNO-A2 catalog, the sky is partitioned into 24 zones of South Polar Distance (SPD), each of width $7^{\circ} .5$. We thereby find 257352 stars spread over 21 zones. For each zone, we plot the residuals in RA and DEC, and keep the 186568 stars within $1 \sigma$ of the mean of each zone. We then compute the relationships between different magnitude systems as shown in Fig. G.1. The corresponding coefficients are provided in Table G.1. $B_{\text {USNO }}$ and $R_{\text {USNO }}$ magnitudes are thus converted into $B_{J}\left(\lambda_{o}^{B}=4400 \AA\right)$ and $R_{C}\left(\lambda_{o}^{R}=\right.$ $6400 \AA)$ magnitudes, which are in turn converted into fluxes with $F_{B, R}=C_{B, R} 10^{-0.4 \times B, R}\left(C_{B}=4.26 \times 10^{-23} \mathrm{~W} \mathrm{~m}^{-2} \mathrm{~Hz}^{-1}\right.$, $\left.C_{R}=3.08 \times 10^{-23} \mathrm{~W} \mathrm{~m}^{-2} \mathrm{~Hz}^{-1}\right)$.

Note that we do not detect any significant bias between northern and southern hemispheres. Systematic effects are observed for $R_{\mathrm{USNO}}$ data for $R_{\mathrm{USNO}}>17.5$ and $\delta<-14^{\circ}$. They will tend to overestimate the contribution of faint stars and are most probably an effect of crowding. 
A.-L. Melchior et al.: The surface brightness of the Galaxy at the solar neighbourhood, Online Material p 13

Table G.1. Coefficients used for the calibration of the USNO magnitudes. In blue $(B), B_{J}=\alpha+\beta\left(B_{\mathrm{USNO}}-15\right)+\gamma\left(B_{\mathrm{USNO}}-15\right)^{2}$. In red $(R)$, $R_{C}=\alpha+\beta\left(R_{\mathrm{USNO}}-15\right)+\gamma\left(R_{\mathrm{USNO}}-15\right)^{2}$. The columns are defined as follows: (1) the zone number, (2) the declination band covered, (3) the filter used, (4) $\alpha$, (5) $\beta$, (6) $\gamma$, (7) the rms error of the polynomial fit, (8) the number of stars effectively used in the fit. We thus used 104354 stars in blue and 154491 stars in red.

\begin{tabular}{|c|c|c|c|c|c|c|c|}
\hline 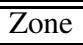 & $\overline{\mathrm{Dec}}$ & Filters & $\bar{\alpha}$ & $\bar{\beta}$ & $\bar{\gamma}$ & $\overline{\text { Residuals }}$ & 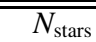 \\
\hline \multirow[t]{2}{*}{075} & $-82.5^{\circ}$ to $-75.0^{\circ}$ & $B$ & 15.24 & 1.14 & 0.0 & 0.38 & 1111 \\
\hline & & $R$ & 15.06 & 1.02 & $6.99 \times 10^{-2}$ & 0.34 & 724 \\
\hline \multirow[t]{2}{*}{150} & $-75.0^{\circ}$ to $-67.5^{\circ}$ & $B$ & 15.59 & 0.95 & $2.32 \times 10^{-2}$ & 0.45 & 2048 \\
\hline & & $R$ & 15.05 & 1.01 & $7.68 \times 10^{-1}$ & 0.42 & 1320 \\
\hline \multirow[t]{2}{*}{225} & $-67.5^{\circ}$ to $-60.0^{\circ}$ & $B$ & 15.80 & 1.00 & $-1.67 \times 10^{-1}$ & 0.67 & 2006 \\
\hline & & $R$ & 14.92 & 0.97 & $6.45 \times 10^{-2}$ & 0.41 & 1653 \\
\hline \multirow{2}{*}{300} & $-60.0^{\circ}$ to $-52.5^{\circ}$ & $B$ & 15.22 & 1.16 & 0.0 & 0.42 & 2509 \\
\hline & & $R$ & 15.04 & 0.95 & $8.09 \times 10^{-2}$ & 0.36 & 2204 \\
\hline \multirow{2}{*}{375} & $-52.5^{\circ}$ to $-45.0^{\circ}$ & $B$ & 15.48 & 1.09 & 0.0 & 0.40 & 3488 \\
\hline & & $R$ & 15.03 & 0.99 & $7.31 \times 10^{-2}$ & 0.38 & 1949 \\
\hline \multirow[t]{2}{*}{450} & $-45.0^{\circ}$ to $-37.5^{\circ}$ & $B$ & 15.27 & 1.15 & 0.0 & 0.36 & 2206 \\
\hline & & $R$ & 15.05 & 0.98 & $5.13 \times 10^{-2}$ & 0.33 & 1220 \\
\hline \multirow[t]{2}{*}{525} & $-37.5^{\circ}$ to $-30.0^{\circ}$ & $B$ & 15.47 & 1.03 & $9.07 \times 10^{-3}$ & 0.50 & 7590 \\
\hline & & $R$ & 14.90 & 0.88 & $8.26 \times 10^{-3}$ & 0.47 & 5061 \\
\hline \multirow[t]{2}{*}{600} & $-30.0^{\circ}$ to $-22.5^{\circ}$ & $B$ & 15.63 & 1.00 & $9.59 \times 10^{-3}$ & 0.48 & 8879 \\
\hline & & $R$ & 15.01 & 0.94 & $4.88 \times 10^{-2}$ & 0.40 & 5771 \\
\hline \multirow[t]{2}{*}{675} & $-22.5^{\circ}$ to $-15.0^{\circ}$ & $B$ & 15.58 & 1.00 & $1.44 \times 10^{-2}$ & 0.42 & 6065 \\
\hline & & $R$ & 14.99 & 0.95 & $3.96 \times 10^{-2}$ & 0.42 & 4578 \\
\hline \multirow[t]{2}{*}{750} & $-15.0^{\circ}$ to $-7.5^{\circ}$ & $B$ & 15.60 & 1.05 & 0.0 & 0.38 & 4196 \\
\hline & & $R$ & 15.16 & 0.92 & $1.21 \times 10^{-2}$ & 0.41 & 6624 \\
\hline \multirow[t]{2}{*}{825} & $-7.5^{\circ}$ to $0.0^{\circ}$ & $B$ & 15.39 & 1.06 & 0.0 & 0.33 & 2470 \\
\hline & & $R$ & 15.07 & 0.97 & 0.0 & 0.34 & 3949 \\
\hline \multirow[t]{2}{*}{900} & $0.0^{\circ}$ to $7.5^{\circ}$ & $B$ & 15.39 & 1.07 & 0.0 & 0.30 & 2171 \\
\hline & & $R$ & 15.09 & 0.99 & 0.0 & 0.33 & 5047 \\
\hline \multirow[t]{2}{*}{975} & $7.5^{\circ}$ to $15.0^{\circ}$ & $B$ & 15.68 & 1.00 & 0.0 & 0.34 & 8428 \\
\hline & & $R$ & 15.13 & 0.97 & 0.0 & 0.33 & 14084 \\
\hline \multirow[t]{2}{*}{1050} & $15.0^{\circ}$ to $22.5^{\circ}$ & $B$ & 15.47 & 0.94 & $2.69 \times 10^{-2}$ & 0.38 & 5945 \\
\hline & & $R$ & 15.11 & 0.95 & $1.68 \times 10^{-2}$ & 0.39 & 11035 \\
\hline \multirow[t]{2}{*}{1125} & $22.5^{\circ}$ to $30.0^{\circ}$ & $B$ & 15.28 & 1.05 & $9.92 \times 10^{-3}$ & 0.33 & 7549 \\
\hline & & $R$ & 15.11 & 0.96 & $1.47 \times 10^{-2}$ & 0.33 & 12974 \\
\hline \multirow[t]{2}{*}{1200} & $30.0^{\circ}$ to $37.5^{\circ}$ & $B$ & 15.21 & 1.13 & $-6.01 \times 10^{-3}$ & 0.28 & 12011 \\
\hline & & $R$ & 15.11 & 0.96 & $8.08 \times 10^{-3}$ & 0.33 & 17769 \\
\hline \multirow[t]{2}{*}{1275} & $37.5^{\circ}$ to $45.0^{\circ}$ & $B$ & 15.40 & 0.99 & $1.68 \times 10^{-2}$ & 0.33 & 12665 \\
\hline & & $R$ & 15.02 & 0.94 & $1.37 \times 10^{-2}$ & 0.39 & 21022 \\
\hline \multirow[t]{2}{*}{1350} & $45.0^{\circ}$ to $52.5^{\circ}$ & $B$ & 15.34 & 1.08 & 0.0 & 0.32 & 9248 \\
\hline & & $R$ & 15.07 & 0.97 & $4.56 \times 10^{-3}$ & 0.39 & 17967 \\
\hline \multirow[t]{2}{*}{1425} & $52.5^{\circ}$ to $60.0^{\circ}$ & $B$ & 15.65 & 1.08 & $-3.69 \times 10^{-2}$ & 0.76 & 3720 \\
\hline & & $R$ & 15.12 & 0.93 & 0.0 & 0.55 & 8983 \\
\hline \multirow[t]{2}{*}{1500} & $60.0^{\circ}$ to $67.5^{\circ}$ & $B$ & 15.23 & 1.15 & $-1.08 \times 10^{-2}$ & 0.31 & 6468 \\
\hline & & $R$ & 15.10 & 0.95 & 0.0 & 0.43 & 9996 \\
\hline \multirow[t]{2}{*}{1575} & $67.5^{\circ}$ to $75.0^{\circ}$ & $B$ & 15.65 & 1.16 & $-2.03 \times 10^{-2}$ & 0.25 & 412 \\
\hline & & $R$ & 14.61 & 1.00 & $2.90 \times 10^{-2}$ & 0.56 & 1031 \\
\hline
\end{tabular}


A.-L. Melchior et al.: The surface brightness of the Galaxy at the solar neighbourhood, Online Material p 14
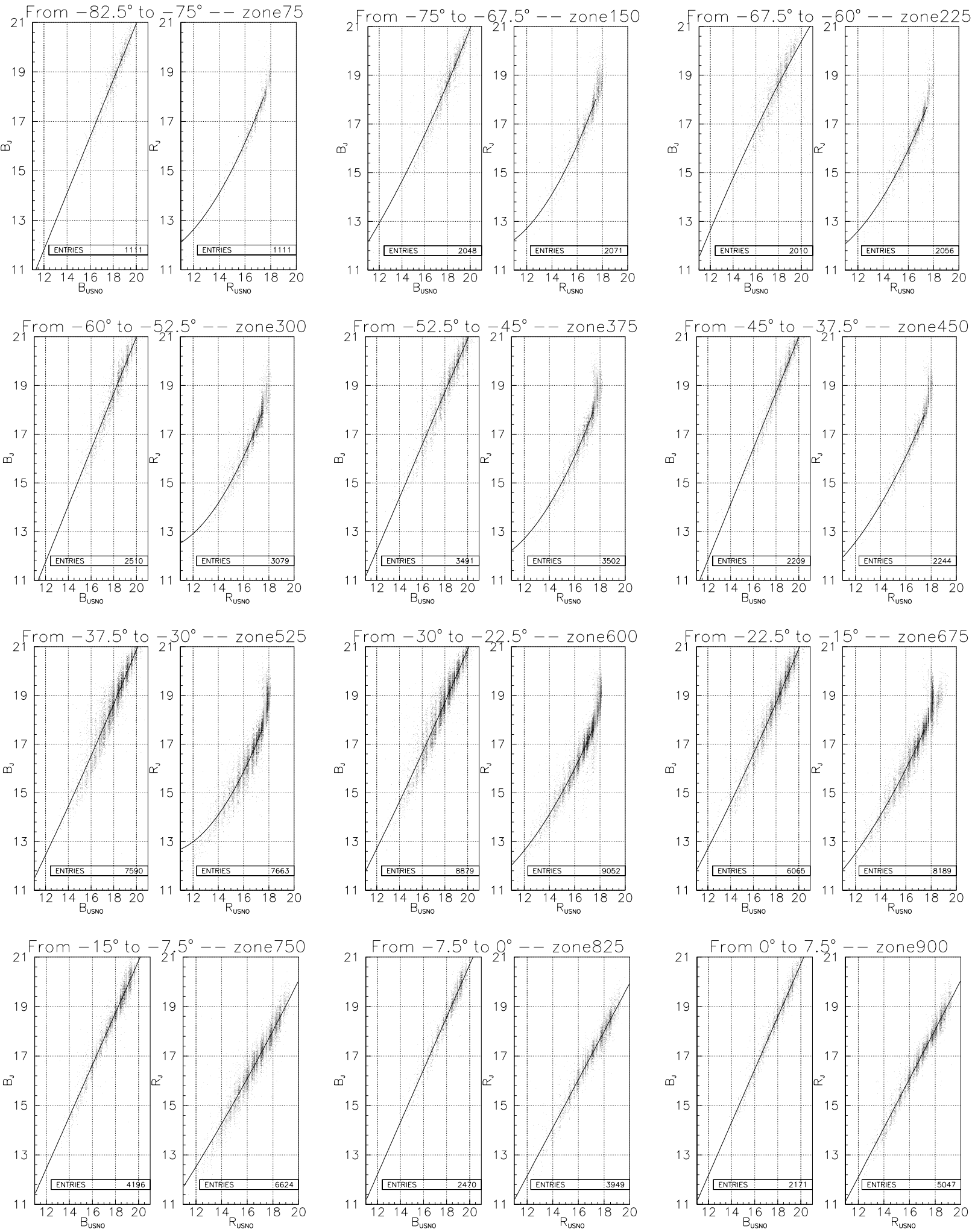

Fig. G.1. Each panel presents the $B_{J}$ versus $B_{\mathrm{USNO}}$ and $R_{C}$ versus $R_{\mathrm{USNO}}$ relations for each South Polar Distance (SPD) interval corresponding to a zone. The polynomial adjustments are superimposed for each relation, and the corresponding coefficients are provided in Table E.1. 
A.-L. Melchior et al.: The surface brightness of the Galaxy at the solar neighbourhood, Online Material p 15
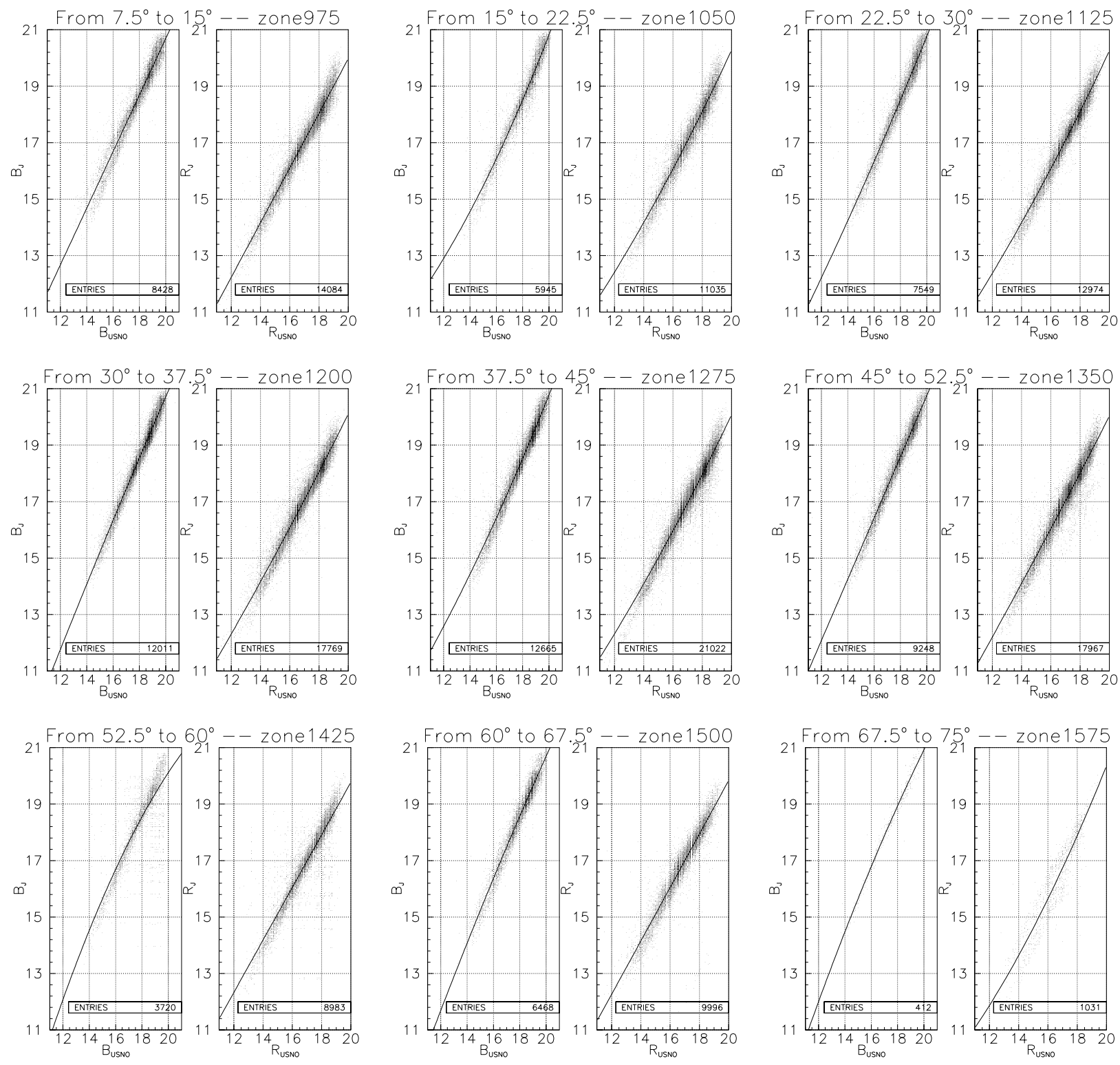

Fig. G.1. continued. 